\title{
Signatures of Photon Localization
}

\author{
A.Z. Genack ${ }^{1}$ and A.A. Chabanov ${ }^{2}$ \\ ${ }^{1}$ Department of Physics, Queens College of the City University of New York, Flushing, NY 11367, USA \\ ${ }^{2}$ Department of Physics and Astronomy, University of Texas, San Antonio, TX 78249, USA
}

(Dated: 10 November 2005)

\begin{abstract}
Signatures of photon localization are observed in a constellation of transport phenomena which reflect the transition from diffusive to localized waves. The dimensionless conductance, $g$, and the ratio of the typical spectral width and spacing of quasimodes, $\delta$, are key indicators of electronic and classical wave localization when inelastic processes are absent. However, these can no longer serve as localization parameters in absorbing samples since the affect of absorption depends upon the length of the trajectories of partial waves traversing the sample, which are superposed to create the scattered field. A robust determination of localization in the presence of absorption is found, however, in steady-state measurements of the statistics of radiation transmitted through random samples. This is captured in a single parameter, the variance of the total transmission normalized to its ensemble average value, which is equal to the degree of intensity correlation of the transmitted wave, $\kappa$. The intertwined effects of localization and absorption can also be disentangled in the time domain since all waves emerging from the sample at a fixed time delay from an exciting pulse, $t$, are suppressed equally by absorption. As a result, the relative weights of partial waves emerging from the sample, and hence the statistics of intensity fluctuations and correlation, and the suppression of propagation by weak localization are not changed by absorption, and manifest the growing impact of weak localization with $t$.
\end{abstract}

PACS numbers: 42.25.Dd, 42.25.Bs, 05.40.-a

\section{OVERVIEW}

Localization $[1,2,3,4,5]$ is inherently a wave interference phenomenon 6] and may therefore occur for both classical 17, 8, 9, 10, 11, 12, 13, 14] and quantum mechanical waves. It may be considered for all manner of excitations including electromagnetic, sound and ultrasound, acoustic and optic phonons, surface plasmons, polaritons, electrons and atoms. This article will discuss signatures of electromagnetic localization in the statistics of radiation reflected from and transmitted through random samples.

The study of transport has been enriched by the many differences and similarities between electronic conduction and electromagnetic wave propagation. Differences in the types of wave and their scattering interactions affect the types of measurements that can be carried out, as well as the possibility and ease with which waves can be localized. Whereas classical waves may be phase-coherent in large static samples since wavelengths greatly exceed atomic dimensions, electronic samples must be cooled to ultra-low temperatures in order to suppress dephasing even in micron-sized samples. The value of the transport mean free path, $\ell$, in which the direction of propagation is randomized, is determined by the specifics of the wave interaction in each material system. However, the character of classical and quantum propagation in mesoscopic systems, in which the wave is temporally coherent throughout the sample [5, 15, 16, 17], is strikingly similar on length scales greater than both the wavelength and the mean free path. For both quantum and classical waves, the character of transport depends upon the closeness of the wave to the threshold of the localization transition separating extended and localized waves.

Ioffe and Regel proposed that propagating waves cannot exist if scattering occurs on a length scale smaller than $\lambda / 2 \pi=1 / k$, in which the amplitude of the wave changes. This suggests that propagation ceases when scattering is strong enough that, $\ell<2 \pi / \lambda$, or $k \ell<1$ [6]. The interference of waves that follow the trajectories in opposite senses around a closed loop enhances the return of the waves and thus suppresses transport. In weakly scattering three-dimensional systems, the probability that a meandering wave trajectory will loop back to a typical coherence volume, $V_{c} \sim(\lambda / 2)^{3}$, is $\sim 1 /(k \ell)^{2}$. The impact of wave interference on average transport is therefore small when $k \ell \gg 1$. The ensemble average of the spread of the square amplitude of the wave is then diffusive and corresponds to the random walk of electrons or photons. However, when $k \ell \sim 1$, the probability that waves will return to a coherence volume is substantial. Interference then impedes the escape from this volume. This consideration leads again to the Ioffe-Regel criterion for localization, $k \ell \sim 1$.

Measurements of $k \ell$ therefore give an indication of the closeness to the localization threshold. Values of $k \ell$ can be determined from measurements of the width of the peak of enhanced backscattering, which is $\sim 1 / k \ell$ [18, 19, 20, 21]. This peak has a triangular cusp with a maximum enhancement of retro-reflection over the diffuse background of a factor of 2 in the diffusive limit [18, 19, 20], and a smaller enhancement due to recurrent scattering events when the localization threshold is approached 21], as seen in Fig. 1. The reflected enhance- 


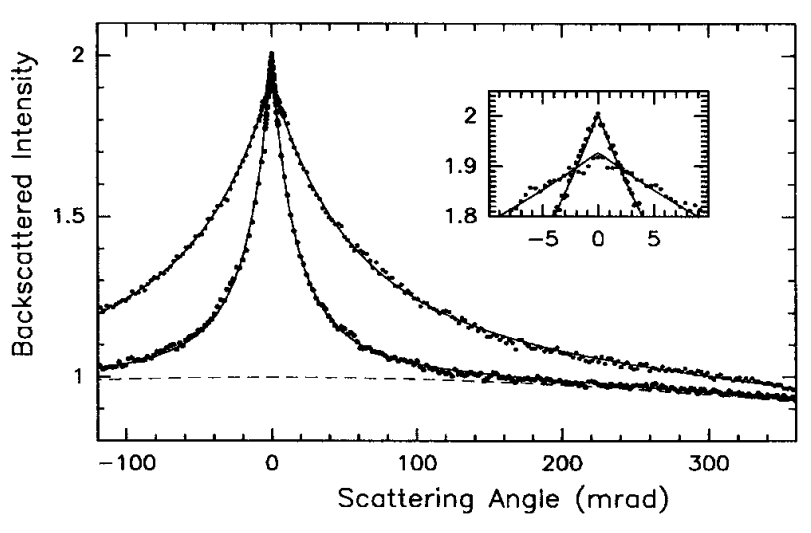

FIG. 1: Coherent backscattering of light measured, with two different values for the transport mean free path $\ell$. The typical angular width varies as $\lambda / \ell$. Narrow cone: a sample of $\mathrm{BaSO}_{4}$ powder with $\ell / \lambda=4$; broad cone: $\mathrm{TiO}_{2}$ sample with $\ell / \lambda=1$. The inset confirms the triangular cusp predicted by diffusion theory, and also shows that the maximum enhancement factor is lowered for the sample with small value of $\ell / \lambda$ [21]. (with kind permission of the authors)

ment peak is the Fourier transform of the point spread function of the incident beam [22].

The closeness to localization in diffusive samples can be ascertained from a determination of $\ell$ from measurements of the scaling of total transmission 23]. Measurements of optical transmission through a wedge-shaped sample of random titania particles dispersed in Polystyrene are shown in Fig. 2 [23]. In the diffusive limit, the ensemble average of the spatial intensity distribution corresponds to the solution of the diffusion equation for the intensity. When absorption is absent, the average energy density falls linearly with depth inside the sample, corresponding to transmission which falls inversely with sample thickness, $L$. When absorption is present in a diffusive sample, the ensemble average of both the energy density versus depth and the transmitted flux versus $L$ falls exponentially with attenuation length, $L_{a}=\sqrt{D \tau_{a}}$, for $L>L_{a}$, where $D$ is the diffusion coefficient and $\tau_{a}$ is the absorption time 23]. The variation of transmission as $1 / L$ is seen in the log-log plot in Fig. 2a for $L<L_{a}$, while the exponential variation of transmission for $L>L_{a}$ is exhibited in the semi-log plot in Fig. 2b. Transmission also falls exponentially as a result of wave localization in strongly scattering samples.

In three-dimensional samples, the diffusion coefficient is given by, $D=\frac{1}{3} v_{E} \ell$, where $v_{E}$ is the transport velocity [24]. The transport velocity is reduced when the wave is resonant with the constituents of the sample, and exhibits dips near Mie resonances with spherical dielectric particles [24, 25, 26, 27]. The transport mean-free-path can be found once $D$ and $v_{E}$ has been determined. The diffusion coefficient can be determined directly from measurements of pulsed transmission [28, 29, 30, 31, 32, 33.
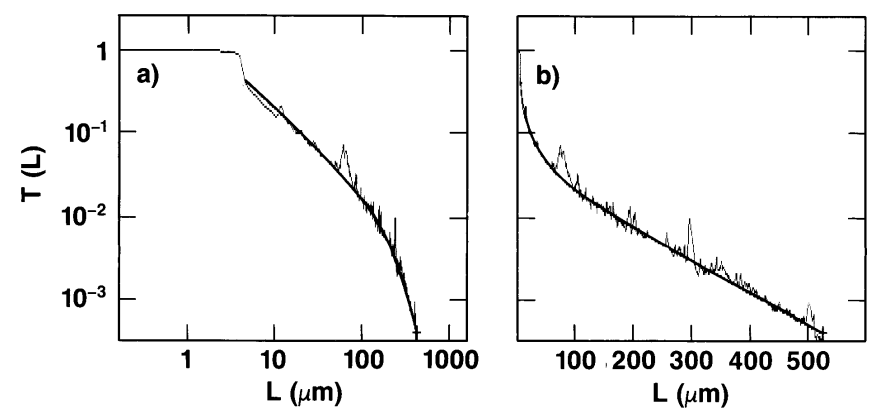

FIG. 2: Scale dependence of optical transmission through a wedge-shaped sample of random titania particles dispersed in Polystyrene. The curve gives the fit of the envelope of the data to diffusion theory. Transmission varies as (a) $1 / L$ for $L<L_{a}$ and as (b) $\exp \left(-L / L_{a}\right)$ for $L>L_{a}=112 \pm 5 \mathrm{~m}$. From Ref. [23].

or from the correlation function of the field or intensity with frequency shift $23,24,29,34,35$. The field correlation function is the Fourier transform of the ensemble average of the response to a short incident pulse [30, 36]. The diffusion coefficient can also be determined in diffusive systems from the intensity correlation function with delay time in a medium in which the constituents are in random motion, such as in colloidal samples [37, 38]. The temporal correlation function reflects the average path length distribution in the medium, which is proportional to the average temporal evolution of transmitted pulses.

For bounded samples, in the absence of inelastic processes, however, any marker of localization must also depend upon the sample geometry, in addition to $\ell$ and $\lambda$, since reflection from the sample boundaries also returns the wave to points within the sample. When inelastic scattering is present, either the impact of inelastic scattering must be explicitly accounted for or a propagation parameter must be found, which still captures an essential aspect of localization and thereby directly expresses the closeness of the wave to the localization threshold.

Spectra of transmitted intensity for extended optical 23] and microwave [39, 40] radiation, such as the microwave measurements shown in Fig. 3a 39], are composed of many overlapping lines which result in spectra without isolated peaks. The wave inside the medium, as well as each of the quasimodes, is extended in space. Within the frequency range in which the wave is localized, however, distinct resonances are observed in the transmission spectrum [2, 41], as seen Fig. 3b. This spectrum of microwave transmission of localized waves was measured in a sample of alumina spheres at low density, contained in a copper tube, which was cooled to the temperature of liquid nitrogen to reduce absorption losses in reflection from the tube. When the wave resonantly excites the sample, its amplitude may be exponentially peaked within the sample [42, 43, 44]. The relatively high intensity in the interior of the sample, as 

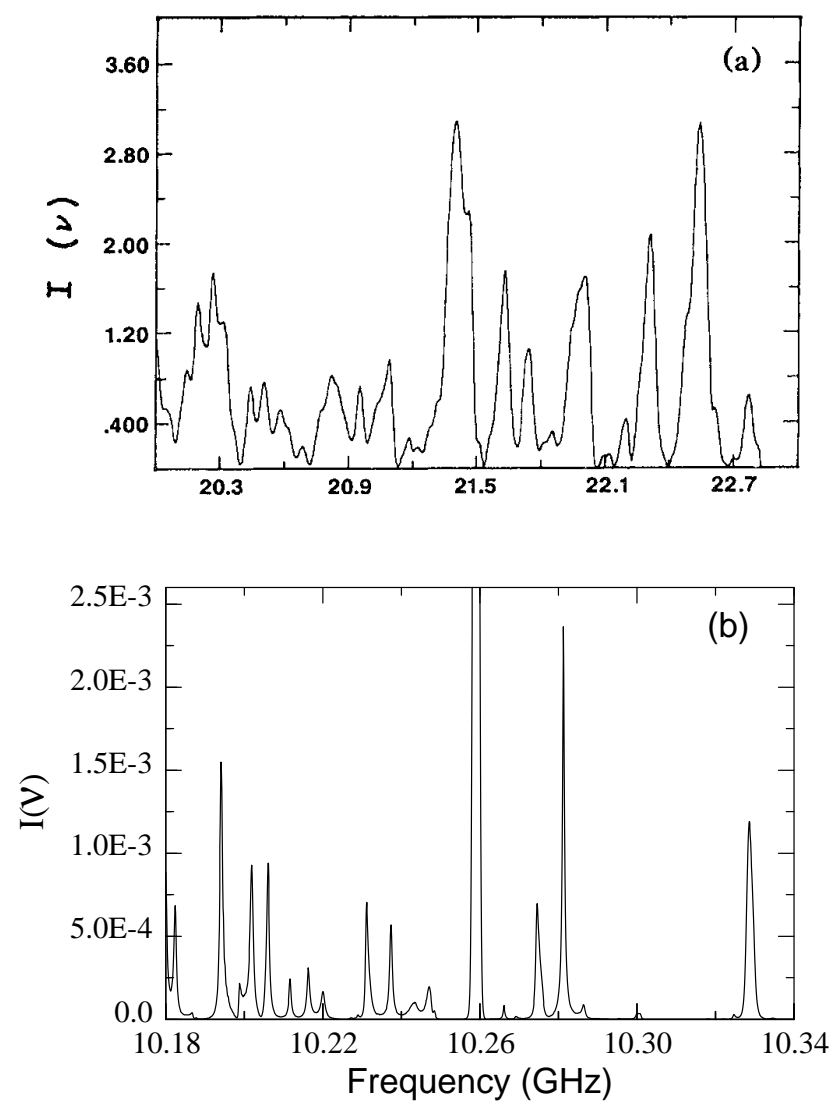

FIG. 3: Isolated peaks in (b), as opposed to (a), are an indication of photon localization. The variance of relative intensity is high in spectra with isolated peaks. From Ref. [39].

compared to near the boundaries, leads to long decay times for the excited mode and, consequently, to distinct narrow spectral lines. Transmission via localized quasimodes is particularly high when intensity is peaked near the middle of the sample 42, 43]. Transmission is also high when two or more quasimodes occasionally overlap spectrally leading to a multi-peaked intensity distribution extending through the sample, known as necklace states [45, 46, 47]. Because these short-lived states are spectrally broad, they dominate average transmission in 1D system. Off resonance, the overall energy density falls exponentially within the sample.

Localizing photons has proven to be a considerable challenge. Photon states bound to subwavelength-scale particles do not exist as they do for electrons bound to ionic cores. In addition, $s$-wave scattering does not exist for photons, as it does for electrons. The electromagnetic scattering strength due to $p$-wave scattering vanishes in the long-wavelength, Rayleigh limit, as $a^{6} / \lambda^{4}$, where $a$ is the particle radius. As a result, it is not possible to enhance scattering by packing many small scattering particles into volumes comparable to the wavelength. Scattering for spherical particles is peaked at Mie resonances, when the particle radius is comparable to $\lambda$. But then it is difficult to create strong scattering with $\ell$ as short as $\lambda$, since the maximum strength of scattering from individual particles is achieved only in dilute systems when scatterers are separated from one another. Because photons are uncharged, however, a pure Anderson localization transition between diffusing and localized waves might occur without the complication of the Coulomb interaction. If this could be observed, it would be possible to determine the critical exponents of the Anderson transition [48, 49]. In addition, the statistics of classical transport can be determined in large ensembles of equivalent samples. To date, photons have only been localized in three-dimensional systems with residual long-range order [41, 50], in one- 51, 52], two- 53, and quasi-1D [41, 55] samples, and in layered samples [56, 57, 58].

John [59] pointed out that waves can be localized in nearly periodic samples in the frequency range of the photonic band gap [60, 61] that forms in the associated periodic structure when the index of modulation is sufficiently large. Though the density of states vanishes in large periodic structures, the introduction of disorder creates localized states in the spectral region of the photonic band gap. The low density of states within the spectral range of the photonic band gap and the narrow, spectrally isolated lines associated with the long residence time of light on resonance with these states lead to spatially localized modes. Measurements of large microwave intensity fluctuations within the low frequency band gap of a cubic wire structure have indicated that localization occurs for intermediate filling fractions of metallic spheres floated within the structure [41, 50].

The relationship between average transmission and fluctuations in transmission lies at the core of the scaling theory of localization [3, 4, 62]. In 1D, it has been hypothesized that the relationship can be expressed via a single parameter [4], which relates fluctuations in transmission, $T$, to the ensemble average of the logarithm of transmission, which is normally distributed. The variance of the logarithm of the transmission in samples of length, $L$, was conjectured to be, $\sigma^{2}=\gamma / L$, where $\gamma$ is the Lyapunov exponent, which is the inverse of the localization length, $\gamma=1 / \xi=-\ln T / L$, for localized waves [4]. Recently strong deviations from the single parameter scaling hypothesis have been predicted in the tail of a band in which the density of states is low [63, 64, 65, 66]. In addition to the localization length, $\xi$, the scaling of transmission then depends upon a second length scale, $\ell_{S}$, whenever $\xi<\ell_{S}$ [64, 67]. This distance is the average spacing between localized states with frequencies between the given frequency and the edge of the band at which the density of states vanishes. The breakdown of single parameter scaling has also been demonstrated in 2D samples 68, 69, 70.

There has been considerable interest in propagation and localization in quasi-1D samples. These samples 
are locally three-dimensional, but have reflecting transverse boundaries with length, $L$, considerably exceeding the sample's transverse dimensions. Since all modes are mixed in such systems, the statistics of propagation are the same at any point on the output surface. Random matrix theory 71,72 is well suited to such systems and has provided nonperturbative results that supplement the findings of diagrammatic perturbation theory in the weak scattering limit [5, 73]. At the same time, measurements of field, intensity, and total transmission in the frequency and time domains can be performed on random ensembles of quasi-1D samples. Because of reflection at the transverse boundaries, the wave is inhibited from straying far from a point in the transverse directions. The number of returns to a typical coherence volume, $V_{c} \sim(\lambda / 2)^{3}$, increases superlinearly, as $L$ increases. As a result, the wave can always be localized as the sample length is increased while the cross sectional area remains fixed. This makes it possible to obtain a comprehensive statistical picture of propagation and localization in samples with well-defined statistical parameters in diffusive and localized samples 74].

Key properties that lend themselves to a universal description are the average values of transmission and conductance and their scaling, and the correlation and variance of local and spatially averaged flux or current. A variety of parameters capture the changing character of diverse aspects of wave propagation in the localization transition. The relationship between these phenomena and the parameters that characterize them are explicit in the absence of inelastic processes, since transport may be described in terms of a single parameter near the center of a band. Indications of a breakdown of single parameter scaling in regions in which the density of states is low have not been found in measurements in locally 3D samples in the slab or quasi-1D geometries. When absorption is present, however, scaling cannot be described in terms of a single parameter, and the resultant exponential reduction in transmission is not related to increasing localization of the wave. Though the conductance can then no longer serve as a localization parameter, measures of correlation and fluctuations of transmitted flux may still be expressed through a single parameter which is a reliable indicator of the nature of wave transport.

We will find that a clearer picture of wave propagation can be achieved once the effects of absorption and weak localization are disentangled. This can be done by transforming from the frequency to the time domain, since absorption has the same impact on all paths at a given delay from an exciting pulse. Since the distribution of paths is not changed by absorption, relative fluctuations are unchanged at a fixed delay time $t$ from an exciting pulse. The temporal evolution of the wave can also be explored by studying its Fourier transform, the field correlation function with frequency shift.

In the absence of inelastic processes, the suppression of transport due to the enhanced return of the wave to a coherence volume may be described in terms of the average value of the dimensionless conductance $g$, which is the scaling parameter [3, 4] of the Anderson localization transition, $g=G /\left(e^{2} h\right)$. In a particular sample realization, the dimensionless conductance equals the transmittance $T$, which is the sum of transmission coefficients over all $N$ input transverse modes, $a$, and outgoing modes, $b, T=\Sigma_{a b} T_{a b}[75,76,77]$. The conductance is naturally measured in electronic experiments since the relative phase of the incident transverse current modes fluctuates randomly over the measurement time to yield the incoherent sum of transmission coefficients. In contrast, studies of classical waves are often carried out with coherent sources, so that it is natural to measure either the field or flux transmission coefficient for a single incident mode to a single outgoing mode or the total transmission which is the transmission coefficient for the net transmitted flux for a single incident transverse mode.

In quasi-1D samples, the number of transverse modes in which two transverse wave polarizations are distinguished is $N=2 \pi A / \lambda^{2}$, where $A$ is the sample cross section. In the diffusive limit, $g \gg 1$, the dimensionless conductance can be approximated by $g=N \ell / L$. This is equivalent to Ohm's law for diffusing particles, since $g=\left(2 \pi \ell / \lambda^{2}\right)(A / L)$. To order $1 / g$, the conductance is reduced from the expression above by a factor of $(1-1 / 3 g)$ 78]. Scaling of average transport is given in terms of the single parameter, $g$, and the localization threshold is reached at $g \approx 1$ [4], at which point $g$ is substantially reduced by renormalization due to weak localization. Since the value of $g$ falls at least as fast as it does for diffusing particles, it is possible to localize waves in static systems by choosing sample lengths greater than the localization length, $\xi=N \ell$. This follows the analysis by Thouless that electrons can be localized in sufficiently long wires when the temperature is low enough that dephasing can be neglected [2].

The relationship of $g$ to localization may be seen from its connection to the Thouless number, $\delta$ [2], which may be expressed as the ratio of the typical width and spacing of resonances of a random ensemble of samples, $\delta=\delta \nu / \Delta \nu$. The level width is the average leakage rate of energy from the electromagnetic quasimodes of the sample and is essentially the inverse of the width of the distribution of photon transit times through the sample when inelastic processes are absent. This corresponds to the width of the field correlation function with frequency shift [79] and is given by $\delta \approx D / L^{2}[2]$. The level spacing is the inverse of the density of states of the sample as a whole, and may be written as $\Delta \nu=1 / n(\nu) A L$, where, $n(\nu)$ is the number of states per unit volume per unit frequency, and $A L$ is the sample volume. The Thouless number is a measure of localization since transport is then suppressed by the poor overlap of quasimodes in different sectors of a sample when, $\delta \nu<\Delta \nu$, and hence, 
$\delta<1$. When $\delta>1$, on the other hand, quasimodes overlap spectrally and transport is relatively uninhibited. Using the Einstein relation for the conductivity, $\sigma=\left(e^{2} / h\right) D n(\nu)$, the conductance can be written as $G=\sigma A / L=\left(e^{2} / h\right) D n(\nu) A / L$. We can then write, $\delta=\delta \nu / \Delta \nu=\left(D / L^{2}\right) /(1 / n(\nu) A L)=\operatorname{Dn}(\nu) A / L=$ $G /\left(e^{2} / h\right)=g[2]$.

The degree of correlation can be identified with $1 / \delta$, since, for monochromatic excitation, the number of quasimodes which are substantially excited is approximately the number of modes within the mode linewidth, which is $\delta \nu / \Delta \nu=\delta$. Since the state of the wave in the sample is approximately specified by the amplitudes of these $\delta$ modes, it may be specified approximately by $\delta$ parameters. As a result, the degree of correlation of intensity is approximately, $1 / \delta$, or equivalently, $1 / g$.

In the diffusive limit, $g \gg 1$, far from the localization threshold, the change in average conductance and the degree of long-range correlation in the flux due to wave interference are both of order $1 / g$. Nonetheless, fluctuations in spatially averaged flux are dramatically enhanced over the values that would be obtained if longrange correlation were absent and the flux in each of the $N$ transmission modes could therefore be assumed to be statistically independent. If this were the case, the variance of total transmission normalized to its ensemble average value, $s_{a}=T_{a} /\left\langle T_{a}\right\rangle$, would be given by $\operatorname{var}\left(s_{a}\right)=1 / N$, while the variance of the conductance normalized to its ensemble average, $s=T / g$, would be given by $\operatorname{var}(s)=1 / N^{2}$, when absorption is absent. Instead, the variance of normalized total transmission over an ensemble is enhanced by a factor of $L / \ell$, to give $\operatorname{var}\left(s_{a}\right) \approx(1 / N)(L / \ell) \approx 1 / g$ 35, 80, 81, 82, 83, 84]. The variance of the normalized dimensionless conductance is enhanced by a factor of $(L / \ell)^{2}$, to give $\operatorname{var}(s) \approx$ $\left(1 / N^{2}\right)(L / \ell)^{2} \approx 1 / g^{2}$ [16, 17, 81]. This leads to a fixed value for the variance of the dimensionless conductance, $\operatorname{var}(T)=g^{2} \operatorname{var}(s) \approx 1$, where, $T=s \times g$. Calculations, in accord with measurements, give $\operatorname{var}(T)=2 / 15$, independent of the size or local scattering strength of the sample [16, 17, 81]. This is termed universal conductance fluctuations.

The neglect of absorption in calculations of propagation makes it possible to highlight specific properties related to weak localization and mesoscopic fluctuations. However, this is only rarely justified in practice. Indeed, the attempt to deal with the reality of absorption has sharpened our understanding of the nature of localization and has provided clear signatures of localization.

\section{ENSEMBLE AVERAGE TRANSMISSION AND REFLECTION}

Early discussions of photon localization were stimulated by the analogy between electron and photon trans- port. The impact of localization on the scaling of transmission was represented as a renormalization of local scattering parameters such as the mean free path or diffusion coefficient 85. It was conjectured that the renormalization of optical transport parameters would be cut off by absorption just as electron renormalization is cut off by dephasing [10]. Microwave measurements of the variation of transmission with thickness in strongly scattering mixtures of metal and dielectric spheres showed departures from diffusive behavior that were interpreted in terms of a scale dependent diffusion coefficient whose value saturated in samples longer than the attenuation length due to absorption [86, 87].

On length scales shorter than the absorption length, the effective diffusion coefficient at the mobility edge is predicted to scale as $1 / L$ and the transmission as $1 / L^{2}$, while the transmission is expected to fall exponentially for localized waves [10, 11]. Such scaling was observed in microwave measurements 86,87 ], but absorption also leads to a more rapid fall-off of transmission in diffusive media [23]. Subsequently, similar scaling was observed in optical measurements in very weakly absorbing and strongly scattering samples of GaAs powders together with a broadening of the coherent backscattering peak [88]. But the role of absorption might still be significant enough to affect these measurements in view of the lengthened dwell time for waves in strongly scattering samples [89]. The closeness to the localization threshold in such systems can be ascertained, however, in a variety of statistical properties of the scattered wave, which will be discussed below.

The interaction of absorption and localization was probed in computer simulations of the temporal variation of the spatial distribution of acoustic energy in an absorbing 2D random sample that is isolated from its surroundings. The vibrational amplitude showed an exponential fall-off with displacement from the single line which was initially vibrating, that persisted in time [90]. This demonstrated that though net energy transport is reduced by absorption, the wave is localized and does not spread throughout the sample. This is understandable since partial waves following a loop in two opposite senses are in phase, when these return to the initial scattering center. Thus the impact of weak localization is undiminished for the surviving partial waves.

The time dependence of flux within a medium can be treated separately from the loss of energy due to dispersed absorption. The statistical impact of absorption at any time can be accounted for by simply multiplying all waves by the factor $\exp \left(-t / \tau_{a}\right)$. This was demonstrated in measurements of field spectra of microwave radiation transmitted through a random ensemble of absorbing dielectric quasi-1D samples [41]. The measured probability distribution of intensity was shown to be in accord with a single-parameter distribution, which is the measured variance of the intensity, as will be discussed 
below. It was further shown that the impact of absorption could be undone in a statistical sense by simply restoring the magnitude of the amplitude of all partial waves emerging from the sample to the value these would have had if absorption were absent without altering the wave. This is done by transforming field spectra into the time domain by multiplying each spectrum by a Gaussian spectrum associated with the Gaussian temporal profile of the incident field amplitude. This product spectrum is then Fourier transformed to give the temporal response to an incident pulse with the prescribed bandwidth. The field in the time domain is then multiplied by $\exp \left(t / 2 \tau_{a}\right)$ and transformed back to the frequency domain. The field transmission coefficient is then obtained by dividing by the Gaussian field spectrum. The complex square of this spectrum is then computed to give the intensity spectrum from which the probability distribution of transmitted intensity is determined. The resultant probability distribution of steady-state intensity was found to be in excellent agreement with the prediction for samples without absorption 41, 73, 91, 92, 93]. The distribution is then given in terms of the single parameter, $g$. This demonstrates that statistically the impact of absorption at any time is simply to reduce the wave amplitude. Absorption thus has a straightforward affect on transport in the time domain, and does not affect weak localization or the associated renormalization of scattering parameters at a specific delay time $t$. This is confirmed by our finding that samples with identical scattering strengths but different values of absorption have the same degree of correlation at a given delay time. Indeed, correlation is not suppressed by the increasing role of absorption for longer time delays, but is rather enhanced with increasing time delay. The reduction of steady-state intensity correlation in the presence of absorption is thus seen to be a result of the decreasing weight of longer paths with increasing absorption. Finally, this analysis shows that the diffusion coefficient is independent of the value of the absorption coefficient.

The role of weak localization increases with time since the probability that a wave trajectory will cross itself increases. We expect therefore that transport will be increasingly suppressed with time even in nominally diffusive samples with $g>1$. An alternate picture of dynamic propagation would be that the initial pulse excites a distribution of quasimodes in the sample. The decay rate of transmission would then decrease in time since the amplitude of longer-lived quasimodes, which are more strongly peaked near the center of the sample, would increase relative to that of shorter-lived resonances. Averaged over an ensemble of random sample realizations, the distribution of leakage rates for quasimodes would be a continuum and would give rise to a continuous reduction of the leakage rate from the sample. Both of these pictures of pulse dynamics are at variance with the particle diffusion picture that gives the ensemble average of intensity as a discrete sum over diffusion modes. The decay rates of the diffusion modes are given by $1 / \tau_{n}=n^{2} \pi^{2} D /\left(L+2 z_{0}\right)^{2}$, where $n$ is a positive integer and $z_{0}$ is the boundary extrapolation length 94, 95, 96]. After a time $\tau_{1}$, the intensity distribution would settle into the lowest diffusion mode and would decay at the rate, $1 / \tau_{1}=\pi^{2} D /\left(L+2 z_{0}\right)^{2}$.

Non-exponential decay has been observed in acoustic scattering in reverberant rooms [97, 98] and solid blocks [99, 100] as well as in microwave scattering in cavities whose underlying ray dynamics is chaotic [101, 102]. The decay rate of electronic conductance has been predicted to fall as a result of the increasing weight of long-lived, narrow linewidth states 103, 104]. The leading correction to the diffusion prediction for the electron survival probability $P_{S}(t)$ was calculated by Mirlin 105 using the supersymmetry approach 106 to be, $-\ln P_{S}(t)=\left(t / \tau_{1}\right)\left(1-t / 2 \pi^{2} g \tau_{1}\right)$.

In pulsed microwave measurements, in quasi-1D random dielectric media composed of random mixtures of low-density alumina spheres, we find a breakdown of the diffusion model in the non-exponential decay of transmission [33]. The decay rate for the transmitted pulses shown in Fig. 4a for different sample ensembles is seen in Fig. $4 \mathrm{~b}$ to decreases in time at a nearly constant rate. A linear fall of the decay rate would be associated with a Gaussian distribution of rates of decay for quasimodes. We find a slightly more rapid decrease of the decay rate which is associated with a slower than Gaussian fall-off of the decay time distribution. The temporal variation of transmission can also be described in terms of the growing the impact of weak localization on the dynamic behavior of waves, which can be expressed via the renormalization of a time-dependent diffusion constant or mean free path [33, 78, 107, 108, 109].

Transmission for localized waves is via resonances with isolated localized states or via spectrally and spatially overlapping states [45, 46, 47, 58]. Steady-state measurements of transmission through localized states have been made in stacks of glass slides 57] and in alternating solid and porous silica layers [58]. Both overlapping and isolated spectral lines are observed. The presence of overlapping lines in the transmission spectrum of a layered material may have a number of causes. When a plane wave enters a layered sample in which isotropic layers are homogeneous, transmission can be described by a $1 \mathrm{D}$ model. When $\xi<L$, then, $\delta<1$, and spectral lines are generally distinct. However, occasional overlap may occur. This has a strong impact on average transmission since the spatial distribution within the sample is quasi-extended and the transmission is consequently high [46, 47]. In samples with transverse inhomogeneity, it is necessary to go beyond a 1D model. Spectral overlap is then produced by broadening associated with the widths of the distributions of the wave vectors in the incident beam and of the structure factor of the layered medium 

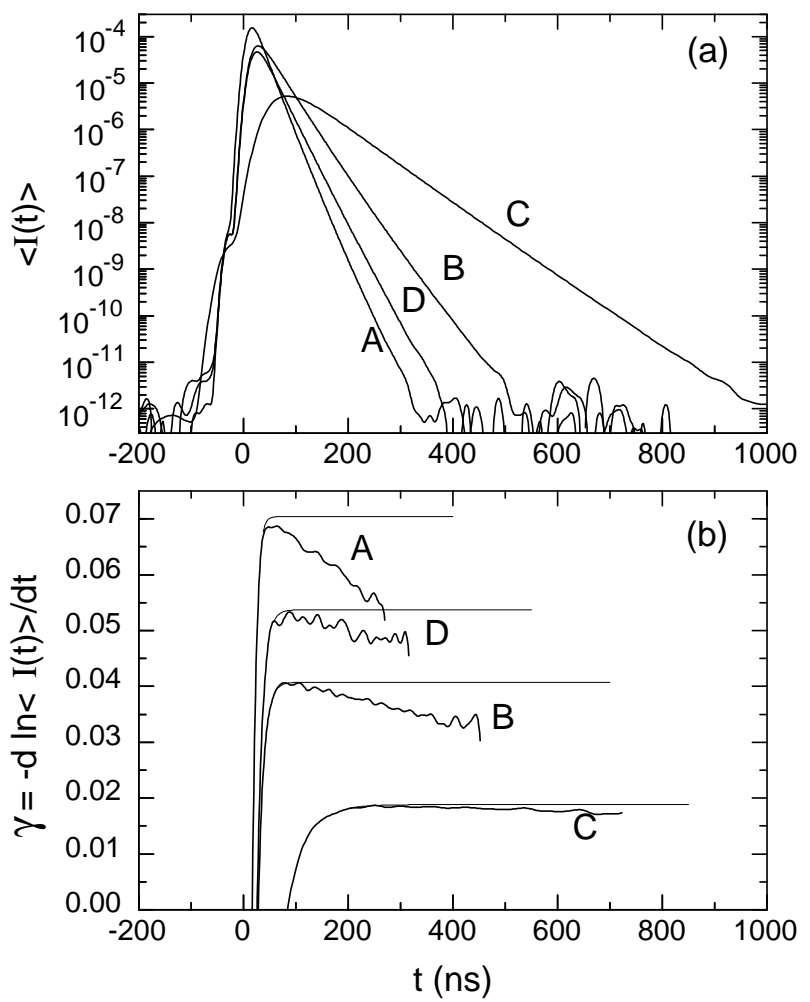

FIG. 4: (a) Average pulsed transmitted intensity in alumina samples of $L=61$ (A), 90 (B and D), and $183 \mathrm{~cm}$ (C); Sample $\mathrm{D}$ is the same as Sample B except for the increased absorption by titanium foil inserted along the length of the sample tube. (b) Temporal derivative of the logarithm of the intensity gives the rate $\gamma$ of the intensity decay due to both leakage out of the sample and absorption. The dashed curves are the decay rates given by the diffusion model. From Ref. [33].

with transverse inhomogeneity. These additional factors give contributions to the $k$-vector distributions which are separately proportional to the inverses of the width of the incident beam and the transverse coherence length of the sample. The overlap of modes is clearly seen in the complex temporal evolution of the pulse in layered porous silica samples [58]. Microwave measurements on a slotted single-mode waveguides packed with random slabs show the impact of overlapping quasimodes within the sample [52].

Lasing is facilitated in localized modes because the excitation is able to penetrate deep into the sample with greatly amplified intensity when the pump laser is resonant with an exponentially localized mode [57]. Light is subsequently emitted into long-lived modes overlapping the region of excitation. This enhances the opportunity for stimulated emission and therefore lowers the laser threshold. In contrast, in diffusive media [110], excitation is restricted to the surface region by multiple scattering and the lasing threshold is not substantially reduced below the threshold for amplified stimu- lated emission in homogeneous media 111]. Localized modes and low threshold lasing were observed in stacks of glass slides with interspersed dye solution or dye-doped plastic sheets [57]. Sharp-line random lasing spectra are also observed in diffusive media 112, 113], in which lasing is initiated in a single or small number of long-lived modes 54, 114, 115, 116]. Lasing also occurs in localized states in nearly periodic anisotropic 1D sample. This is observed in disordered cholesteric liquid crystals. In the ordered structures, the director pointed along the average molecular axis in each layer rotates with well-defined pitch. A distinctive progression of band edge modes is then observed. But in liquid crystals in which periodicity has been disrupted to a lesser or greater extent, localized states form at the band edge. Lasing then occurs in these states 117, 118]. Lasing is also observed when a localized state is introduced into the band gap by a discontinuous jump in the angle of the director [119, 120].

\section{STEADY-STATE STATISTICS}

In the absence of absorption, there is an explicit connection between average transport and mesoscopic fluctuations and correlation in quasi-1D samples since these can all be expressed in terms of a single parameter, $g$. Since this direct link between the averaged transmission and the statistics of transmission is lost in absorbing samples, it is worthwhile to consider the statistics of relative fluctuations. We consider the probability distributions of transmitted intensity and total transmission normalized by their ensemble averages, $s_{a b}=T_{a b} /\left\langle T_{a b}\right\rangle$ and $s_{a}=T_{a} /\left\langle T_{a}\right\rangle$, respectively. The relationship between the probability distributions of intensity and total transmission was found by Kogan and Kaveh from random matrix theory [91],

$$
P\left(s_{a b}\right)=\int_{0}^{\infty} \frac{d s_{a}}{s_{a}} P\left(s_{a}\right) \exp \left(-s_{a b} / s_{a}\right) .
$$

This is equivalent to the relationship between the moments of the distributions of $s_{a}$ and $s_{a b}$,

$$
\left\langle s_{a b}^{n}\right\rangle=n !\left\langle s_{a}^{n}\right\rangle,
$$

for the $n$-th moment 91]. From the relationship between the second moments, obtained from Eq. (2) for $n=2$, we find, $\operatorname{var}\left(s_{a b}\right)=2 \operatorname{var}\left(s_{a}\right)+1$. In the limit of weak scattering and vanishing absorption, the distribution of total transmission is found from diagrammatic and from random matrix theory to be [91, 92],

$$
P\left(s_{a}\right)=\int_{-i \infty}^{i \infty} \frac{d p}{2 \pi i} \exp \left[p s_{a}-\Phi(p)\right],
$$

where,

$$
\Phi(p)=g \ln ^{2}(\sqrt{1+p / g}+\sqrt{p / g}) .
$$


This leads to a connection between fluctuations and average transmission, $\operatorname{var}\left(s_{a}\right)=2 / 3 g$. Since the probability distributions of intensity and total transmission are given in terms of the single parameter, $g$, in this limit, these distributions may equally be expressed as functions only of $\operatorname{var}\left(s_{a}\right)$.

The distributions of $s_{a}$ and $s_{a b}$ in three quasi-1D samples composed of randomly positioned Polystyrene spheres with different diameters and lengths in the frequency range $16.8-17.8 \mathrm{GHz}$, in which the variation in the mean free path is small, are shown in Fig. 5 [93, 121]. The distributions broaden, and the deviation from a Gaussian become more pronounced, as either the sample length increases or the cross-sectional area decreases. The results shown in Fig. 5 are for samples, $a, b$, and $c$, which would have values of the dimensionless conductance in the absence of localization corrections and absorption, $g=N \ell / L$, of 15.0, 9.0, and 2.25, respectively 93]. The straight line in Fig. 5b is a plot of $\exp \left(-s_{a b}\right)$, which is the prediction of Rayleigh for the intensity distribution for one polarization component of the field in a sample in which the number of transverse modes, $N$, diverges and in which long-range correlation vanishes. The distribution of total transmission in the diffusive limit is Gaussian with variance $2 / 3 g$. This is in contrast to an estimated value of $1 / N$, if long-range correlation were neglected.

The theoretical expressions for the full distribution functions in Eqs. (1) and (3) are given as functions of $g$ for nonabsorbing samples. We now consider the full transmission distributions in the presence of absorption. Since the decreased value of $g$ due to absorption does not lead to enhanced values of intensity correlation but rather the reverse, it is not possible to utilize the value of $g$ in Eqs. (1) and (3). But since $\operatorname{var}\left(s_{a}\right)=2 / 3 g, P\left(s_{a}\right)$ may be expressed as a function of $\operatorname{var}\left(s_{a}\right)$. When $\operatorname{var}\left(s_{a}\right)$ is calculated for the three samples in Fig. 5, and 2/3var $\left(s_{a}\right)$ is substituted for $g$ in Eq. (4), the resulting curves plotted in the figure are in excellent agreement with the measured distributions. The measured values of $2 / 3 \operatorname{var}\left(s_{a}\right)$ may be compared to the values of $g$ calculated without absorption which were given above: 10.2 for sample $b$ instead of 9 , and 3.06 for sample $c$ instead of 2.25 . The measured values of $2 / 3 \operatorname{var}\left(s_{a}\right)$ are larger when absorption is present, because absorption reduces the weight of longer paths. The distribution of Eq. (4) gives the exponential tail $P\left(s_{a}\right) \sim \exp \left[-2 s_{a} / 3 \operatorname{var}\left(s_{a}\right)\right]$ 91, 92]. These results are in excellent agreement with measurements [93].

We find that $\operatorname{var}\left(s_{a}\right)$ does not drop sharply with increasing absorption as does the average transmission, but is only weakly suppressed [41]. This indicates that the broad distribution of intensity and of total transmission, which is characteristic of wave propagation in random systems, is preserved in absorbing media. It is of interest therefore to examine the scaling of the statistics of propagation. The scaling of $\operatorname{var}\left(s_{a}\right)$ determined at a number of frequencies is shown in Fig. 6 [41]. $\operatorname{Var}\left(s_{a}\right)$ increases
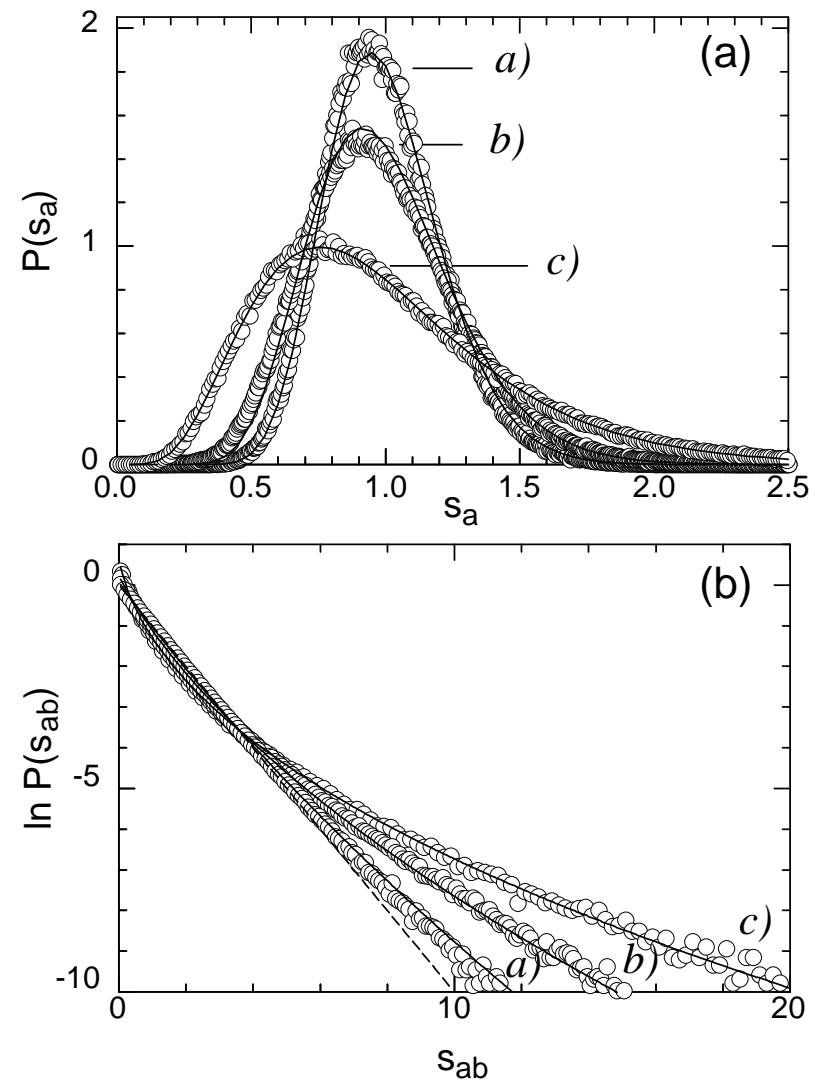

FIG. 5: Distribution functions of (a) the normalized total transmission and (b) transmitted intensity, $P\left(s_{a}\right)$ and $P\left(s_{a b}\right)$, respectively, for three Polystyrene samples with dimensions: a) $d=7.5 \mathrm{~cm}, L=66.7 \mathrm{~cm} ; b) d=5.0 \mathrm{~cm}, L=50.0 \mathrm{~cm} ; \mathrm{c}$ ) $d=5.0 \mathrm{~cm}, L=200.0 \mathrm{~cm}$. Solid lines represent theoretical results obtained from Eqs. (1) and (3), with measured values of $2 / 3 \operatorname{var}\left(s_{a}\right)$ substituted for $g$. The dashed line in (b) is a semi-logarithmic plot of the Rayleigh distribution, $P\left(s_{a b}\right)=$ $\exp \left(-s_{a b}\right)$. From Refs. [93, 121].

exponentially once it becomes of the order of unity. This is expected for a localization parameter, since, in the absence of absorption, $\operatorname{var}\left(s_{a}\right) \sim 1$, when $g \sim 1$. The availability of a measurable localization parameter makes it possible to definitively index the nature of propagation via a parameter marking the closeness to the localization threshold. The measurement of $\operatorname{var}\left(s_{a}\right)$ makes it possible to probe the array of factors that foster localization, including size, concentration, and structural correlation.

\section{WINDOW OF LOCALIZATION}

In the absence of absorption, localization can always be brought about by increasing the length of a sample, while holdings its cross-sectional area fixed 2]. To induce localization in as short a sample as possible, strongly 


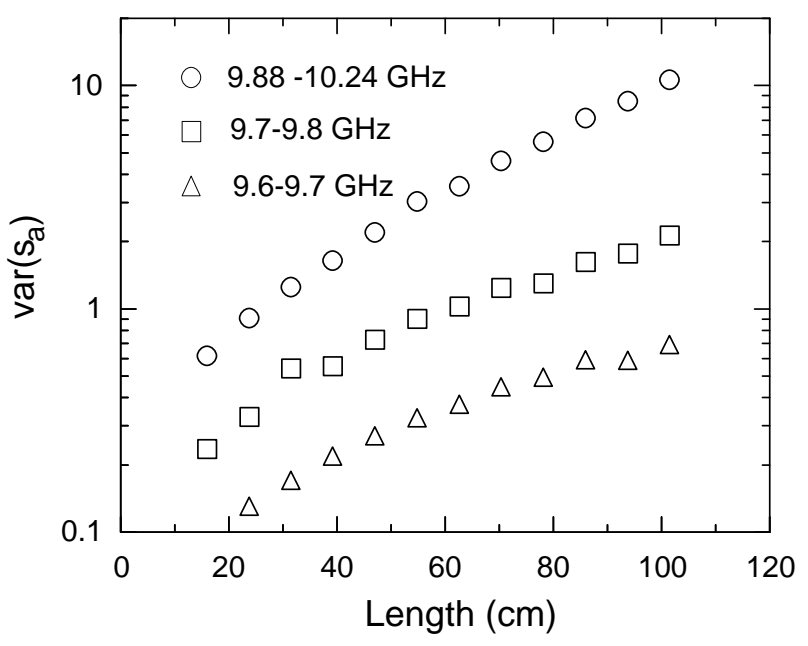

FIG. 6: Scaling of $\operatorname{var}\left(s_{a}\right)$ in samples of alumina spheres. Above a value of the order of unity, $\operatorname{var}\left(s_{a}\right)$ increases exponentially. From Ref. 41].

scattering samples should be used. Since the strength of scattering is peaked near Mie resonances, it is useful to carry out measurements in samples containing spherical scatterers at wavelengths comparable to the diameter of the spheres. In order for the resonances not to be washed out by correlated scattering among neighboring spheres, samples with low density of spheres were studied. Microwave measurements were made in quasi$1 \mathrm{D}$ samples of alumina spheres at a volume fraction of $f=0.068[55,122$. The alumina spheres have a diameter of $0.95 \mathrm{~cm}$ and an index of refraction of $n=3.14$. These are placed at the centers of 1.9-cm-diameter Styrofoam spheres. The sample is contained in a 7.3 -cm-diameter copper tube with sample lengths up to $L=90 \mathrm{~cm}$. An ensemble of 5,000 random sample configurations is created by rotating the tube between subsequent field spectrum measurements. For each sample configuration, the field spectrum yields the corresponding frequency variation of the transmitted intensity $I_{a b}$. The average intensity $\left\langle I_{a b}\right\rangle$ for $L=80 \mathrm{~cm}$ samples, shown in Fig. 7a, exhibits distinct drops near Mie resonances. The resonant character of scattering is further indicated by the sharp peaks in the average photon transit time, $\langle\tau\rangle$, seen in Fig. $7 \mathrm{~b}$. The transit time or single-channel delay time is given by $\tau_{a b}=s_{a b} d \phi_{a b} / d \omega$, where $\phi_{a b}$ is the phase accumulated by the field as it propagates through the sample, from incident channel $a$ to outgoing channel $b$, and $\omega$ is the angular frequency [123, 124]. Since low transmission can be due to absorption and long dwell time can be associated with microstructure resonances 24, 25, 26], the average transmission and dwell time do not provide definitive measures of the closeness to the localization threshold. This can be obtained, however, from the measurement of $\operatorname{var}\left(s_{a b}\right)$, shown in Fig. 7c. For diffusive waves obeying Rayleigh
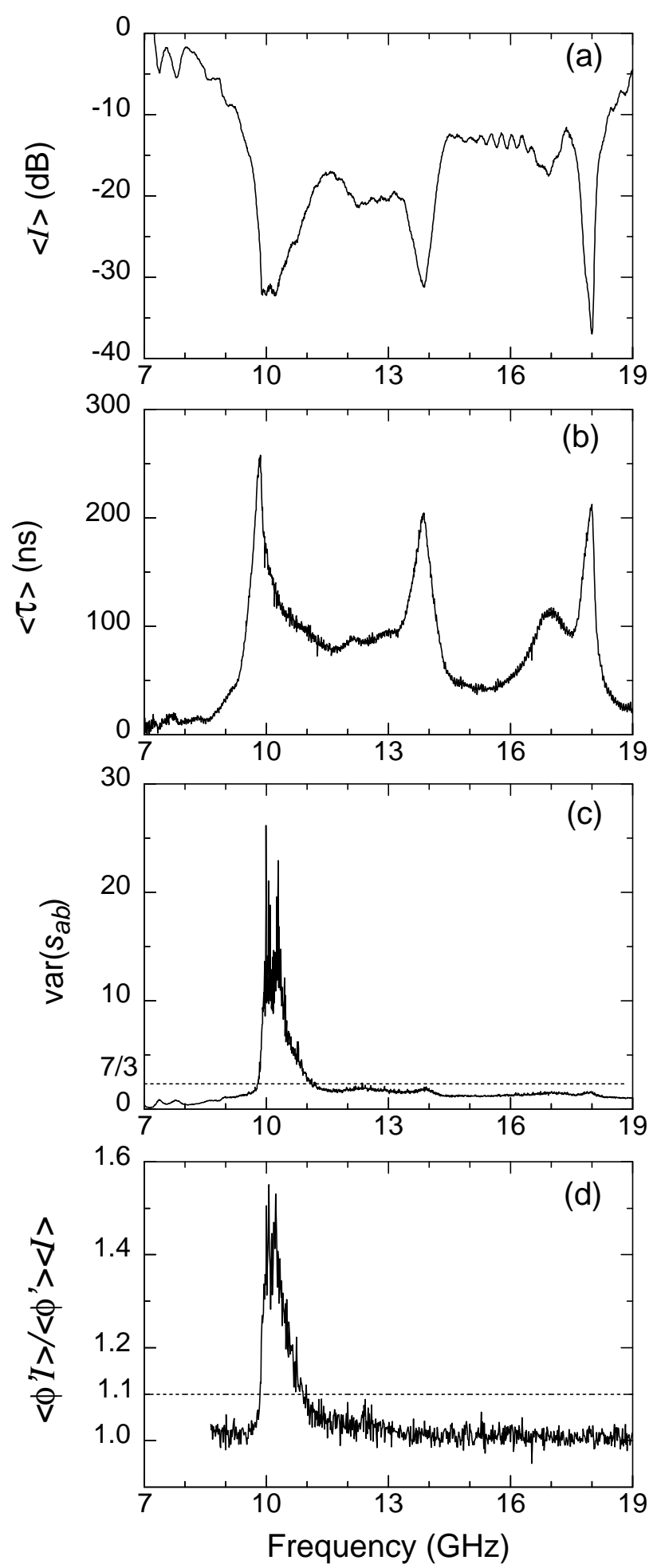

FIG. 7: (a) Average transmitted intensity, $\langle I\rangle$, (b) average photon transit time, $\langle\tau\rangle$, (c) variance of normalized transmitted intensity, $\operatorname{var}\left(s_{a b}\right)$, and (d) dimensionless ratio, $\left\langle\hat{\phi}^{\prime} \hat{I}\right\rangle=\left\langle\phi^{\prime} I\right\rangle /\left\langle\phi^{\prime}\right\rangle\langle I\rangle$, versus frequency in a quasi-1D alumina sample with $L=80 \mathrm{~cm}$ and alumina volume filling fraction $f=0.068$. The dashed lines in (c) and (d) indicate the localization threshold. From Refs. [55, 134]. 
statistics, $\operatorname{var}\left(s_{a b}\right)=1$. Lower values of $\operatorname{var}\left(s_{a b}\right)$, seen below $8.5 \mathrm{GHz}$, indicate a significant ballistic component in the transmitted field, whereas higher values indicate the presence of substantial long-range correlation. The horizontal dashed line in Fig. $7 \mathrm{c}$ represents the localization threshold, $\operatorname{var}\left(s_{a b}\right)=7 / 3$, which corresponds to $\operatorname{var}\left(s_{a}\right)=2 / 3$. In alumina samples with $L=80 \mathrm{~cm}$, the localization threshold is crossed in a narrow frequency range above the first Mie resonance. Large fluctuations of intensity reflected in measurements of $\operatorname{var}\left(s_{a b}\right)$ above the localization threshold, seen in Fig. 7c, are a result of the greatly enhanced tail of the intensity distribution for localized radiation associated with the strong difference in transmission when a sample is on or off resonance. Within the localization window, $\operatorname{var}\left(s_{a b}\right)>7 / 3$, the probability distribution for intensity becomes extraordinarily broad [41].

The strength of scattering cannot be substantially increased by increasing the concentration of randomly positioned alumina spheres. When the density of alumina spheres is increased individual sphere resonances are washed out. This is shown in Fig. 8a in measurements of $D$ as a function of volume filling fraction, $f$, of nearly spherical alumina particles with approximate diameter $0.95 \mathrm{~cm}$ and index of refraction $n=2.95$ [27]. Measurements of the mean free path for $f=0.30$ are shown in Fig. 8b 125]. These results yield the transport velocity shown in Fig. 8c, which is substantially reduced over a broad spectral range in agreement with CPA calculations [126].

\section{DELAY TIME STATISTICS}

In addition to the statistics of steady-state transmission, there exists a rich statistics of dynamics, which is strongly correlated to static aspects of propagation 103, 127, 128. For a spectrally narrow pulse, the single-channel delay time is found to be the derivative of the phase of the transmitted field with frequency, $\tau_{a b}=s_{a b} d \phi_{a b} / d \omega \equiv \phi^{\prime}[123,124]$. The single-channel delay time can similarly be defined for the reflected field. The interplay between the single-channel delay time and transmission coefficient is expressed in the joint probability distribution $P\left(\phi^{\prime}, I\right)$. The phase is discontinuous with a jump of $\pi$ radians, as the null in intensity in the speckle pattern is traversed [129], giving rise to strong fluctuations in $\phi^{\prime}$ at low intensities. For a given value of intensity $I$ the delay time $\phi^{\prime}$ is normally distributed with normalized standard deviation, $\Delta \phi^{\prime} /\left\langle\phi^{\prime}\right\rangle=\sqrt{Q / 2 I}$, whereas the second-order cumulant correlator vanishes, $\left\langle\phi^{\prime} I\right\rangle-\left\langle\phi^{\prime}\right\rangle\langle I\rangle=0$. For, $\ell<L<\xi$, in the diffusive regime, in the absence of absorption, the probability distribution of the delay times is $P\left(\hat{\phi}^{\prime} \equiv \phi^{\prime} /\left\langle\phi^{\prime}\right\rangle\right)=$ $(Q / 2)\left[Q+\left(\hat{\phi}^{\prime}-1\right)^{2}\right]^{-3 / 2}$, where $Q \approx 1$ in transmission [130, 131] and $Q \approx(L / \ell)^{2}$ in reflection 130, 131, 132].
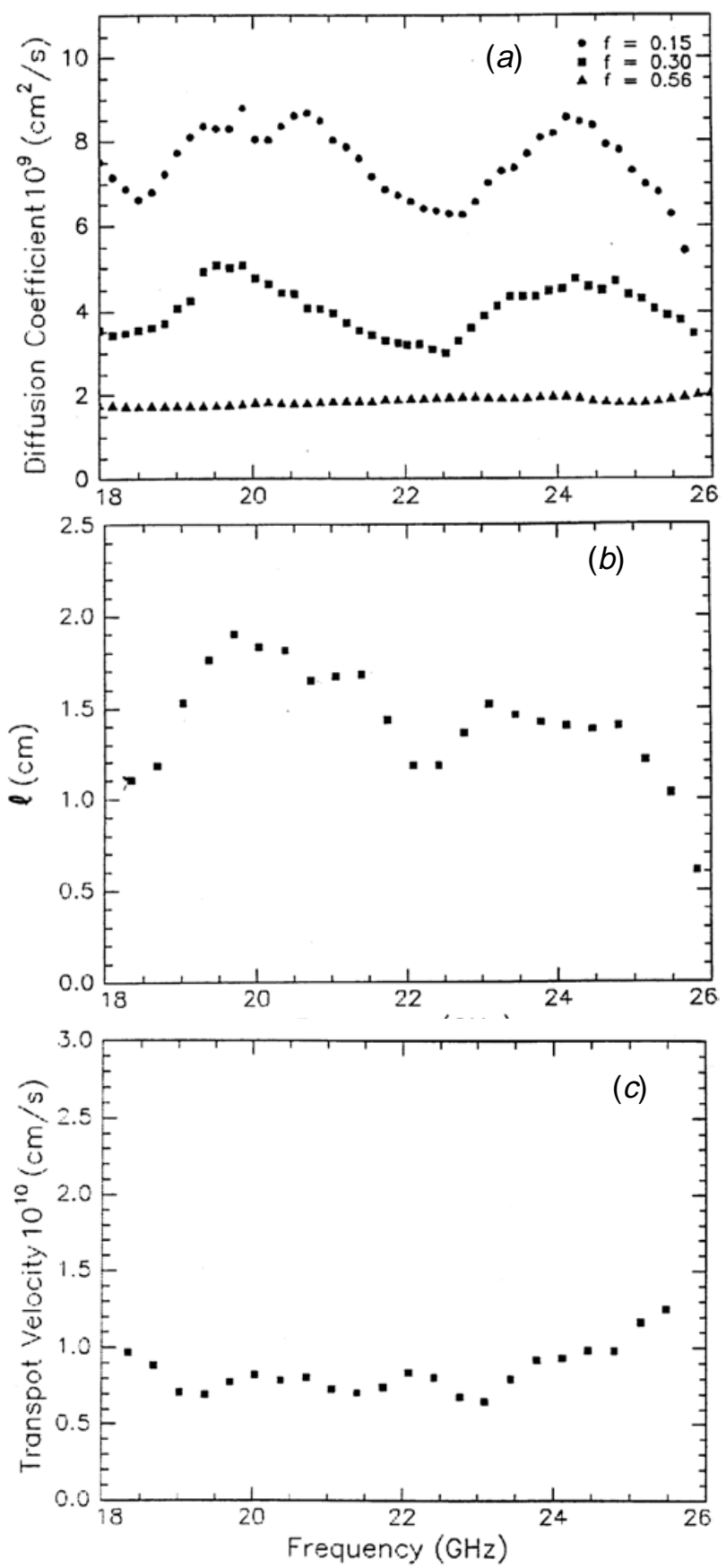

FIG. 8: (a) Frequency dependence of the diffusion coefficient for mixtures of industrial-grade 0.95 -cm-diameter alumina spheres within hollow polypropylene spheres for various alumina filling fractions, $f$; (b) Transport mean free path for sample with $f=0.30$; (c) Transport velocity for $f=0.30$ obtained from (a) and (b), $v_{E}=3 D / \ell$. From Refs. 27, 125].

Absorption can be accounted for simply by a change in $Q$. This agrees well with microwave [130, 131] and optical [133] measurements.

The statistics of dynamics of localized waves differ fundamentally from those of diffusive waves since the trans- 
mission spectrum includes narrow spikes, since $\delta<1$. Long delay times for localized waves occur at peaks in transmission, associated with resonant tunneling through localized states, so that $\left\langle\phi^{\prime} I\right\rangle-\left\langle\phi^{\prime}\right\rangle\langle I\rangle>0$. The correlator between $\phi^{\prime}$ and $I$ thus provides a dynamical test of photon localization. The frequency variation of the ratio $\left\langle\hat{\phi}^{\prime} \hat{I}\right\rangle=\left\langle\phi^{\prime} I\right\rangle /\left\langle\phi^{\prime}\right\rangle\langle I\rangle$ in a quasi-1D sample of alumina spheres with $L=80 \mathrm{~cm}$ is plotted in Fig. $7 \mathrm{~d}$ 134]. It is unity in the diffusive limit and rises above unity as localization is approached. $\left\langle\hat{\phi}^{\prime} \hat{I}\right\rangle$ and $\operatorname{var}\left(s_{a}\right)$ both rise appreciably only within the localization window in Fig. 7. The localization threshold, at which $\operatorname{var}\left(s_{a b}\right)=7 / 3$, corresponds to the condition $\left\langle\hat{\phi}^{\prime} \hat{I}\right\rangle=1.1$, shown as the dotted line. The conditional probability distribution $P_{I}\left(\hat{\phi}^{\prime}\right)$ given $I$ for localized waves in transmission is found to exhibit an exponential fall-off, with an asymmetry in the distribution, which increases with decreasing $I$, and to have a normalized standard deviation of $\Delta \phi^{\prime} /\left\langle\phi^{\prime}\right\rangle \propto I^{-0.25}$ 134. The distribution, $P\left(\phi^{\prime}\right)$, of the single-channel delay time is given by random-matrix theory [132]. In the localized regime, it is found to have a universal quadratic tail, $P\left(\phi^{\prime}\right) \propto\left|\phi^{\prime}\right|^{-2}$ [135, 136, 137]. In samples of the finite length $L$, this algebraic tail eventually crosses over to a log-normal tail, at exponentially long delay times, $\left|\phi^{\prime}\right|>\tau_{s} \exp (L / \xi)$, where $\tau_{s}$ is the scattering time 104, 105, 138, 139]. There is an interesting dynamic signature of localization in reflection: the distribution $P\left(\phi^{\prime}\right)$ differs in the two cases, $a=a^{\prime}$ and $a \neq a^{\prime}$. The maximal value of $P\left(\phi^{\prime}\right)$ in the case $a=a^{\prime}$ is greater than that for $a \neq a^{\prime}$ by a factor close to $\sqrt{2}$ [132]. The effect appears only in the localized regime, as shown in Fig. 9. While there is a complete theory of the statistics of dynamics in reflection, in transmission only the 1D case, $N=1$, has been solved exactly [140]. In this case, the transmission delay time is found to be the mean of reflection delay times of the both ends of the waveguide. For $N>1$, an approximate solution was found [140], which is shown in Fig. 10a, and compared to results of numerical simulations. Microwave measurements of $P\left(\phi^{\prime}\right)$ within the localization window were carried out in ensembles of alumina samples of increasing length [134]. The distributions of the normalized single-channel delay time in samples with $L=25$ and $90 \mathrm{~cm}$ are shown in Fig. 10b. The values of $\operatorname{var}\left(s_{a}\right)$ in these samples are 1.0 and 7.1, and the ensemble-averaged values of $\phi^{\prime}$ are $\left\langle\phi^{\prime}\right\rangle=21$ and 122 ns, respectively. As Fig. 10b suggests, $P\left(\phi^{\prime}\right)$ becomes more asymmetric as $L$ increases, and reaches its peak at values of $\hat{\phi}^{\prime}$ further below its average value of unity. The measured distributions of Fig. 10b appear to be similar to the calculated distributions in Fig. 10a, though a closer comparison reveals a discrepancy in the tail that may be due to the presence of absorption in alumina samples.

The averaged delay time is found by summing the single-channel delay time weighted by the intensity, $W_{a b}=I_{a b} \phi_{a b}^{\prime}$. When summed over all input and out-
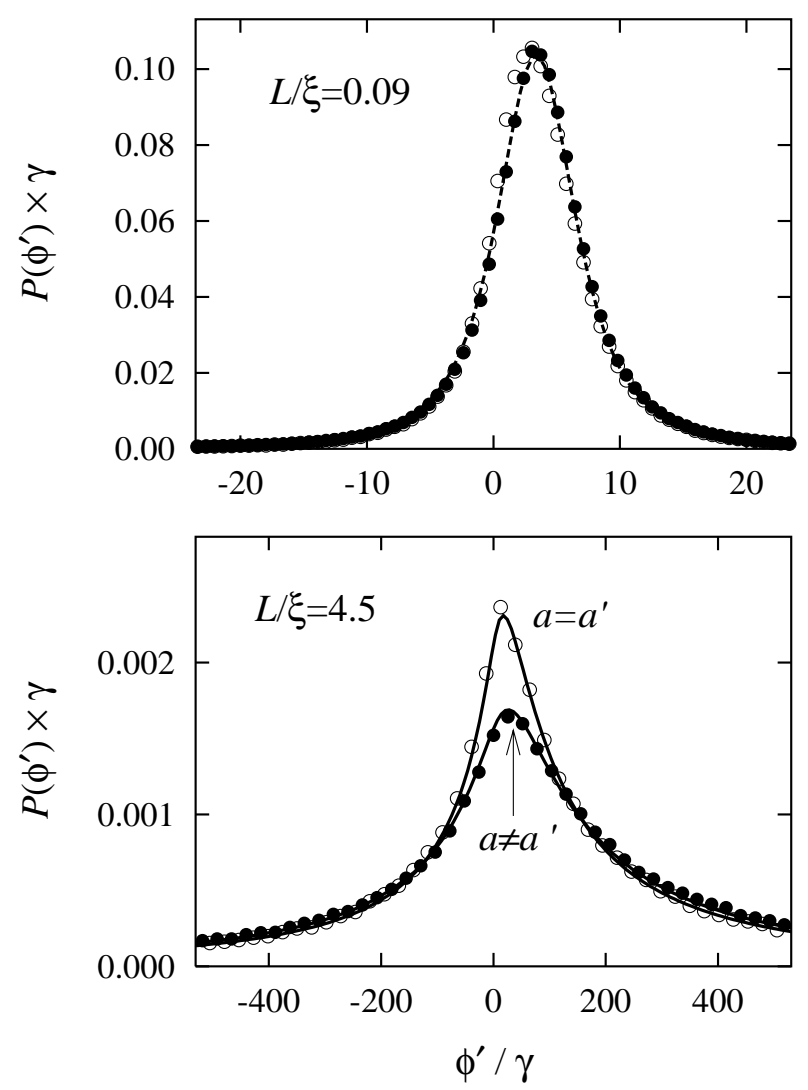

FIG. 9: Calculations of distribution of the single-channel reflection delay time in the diffusion (top panel) and localized (bottom panel) regimes [132]. The maximal value of $P\left(\phi^{\prime}\right)$ in the case $a=a^{\prime}$ of equal excitation and reflection modes (open circles) is greater than that for $a \neq a^{\prime}$ (solid circles) by a factor close to $\sqrt{2}$. The effect appears only in the localized regime. (with kind permission of the authors)

put modes, $W_{a b}$ relates directly to a fundamental dynamic quantity in condense matter, namely the number of microstates per unit frequency interval $d \omega$ inside the scattering medium, $N(\omega)$, 141

$$
\frac{1}{\pi} \Sigma_{a b}^{2 N} I_{a b} \phi_{a b}^{\prime}=N(\omega) .
$$

The summation runs over $N$ channels in both reflection and transmission. Though the density of states in an open (scattering) system is ill-defined, the l.h.s. of the Eq. (5) is well defined and proportional to $\int d r\left(\left|\psi_{\omega}(r)\right|^{2}-1\right), 141 \mid$ which equals the stored electromagnetic energy [142]. In the diffusive limit of Gaussian field statistics, the distribution of $W=W_{a b}$ is a doublesided exponential 130, 131]. In a 1D sample with $L>\xi$, an algebraic decay with exponent $-4 / 3$ was calculated for $P(W)$ using a model of resonant transport via localized states [136]. The probability distribution of the normalized weighted delay time, $P(\hat{W} \equiv W /\langle W\rangle)$, for localized waves in a quasi-1D alumina samples with $L=90$ $\mathrm{cm}$ is shown in Fig. 11 [134]. The distribution $P(\hat{W})$ is 

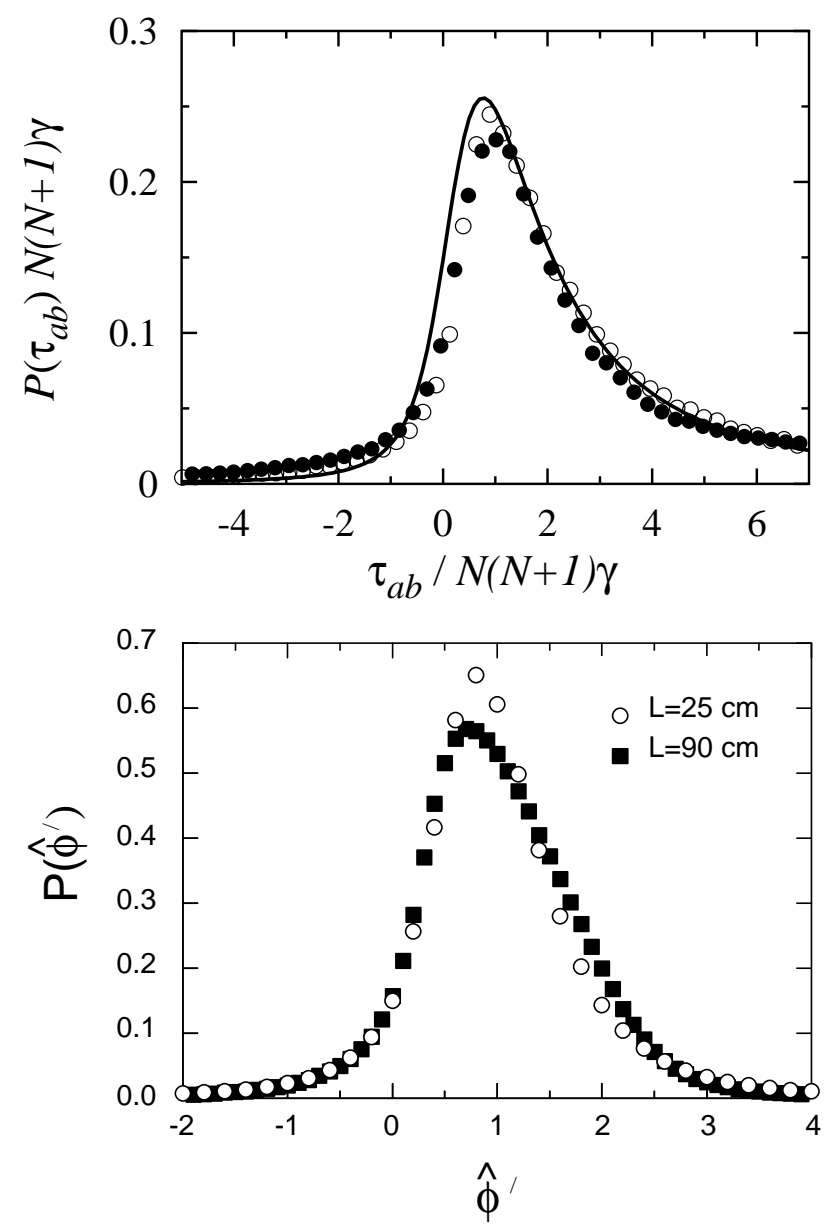

FIG. 10: (a) Distribution of the single-channel transmission delay time obtained from numerical simulations of random scattering in planar waveguides with $N=2$ (open circles) and $N=30$ (filled circles) channels. The solid line is the analytical prediction, independent of $N$ [140]. (with kind permission of the author); (b) Probability distribution of the normalized delay time, $P\left(\hat{\phi}^{\prime}\right)$, in alumina samples with $L=25$ (circles) and $90 \mathrm{~cm}$ (squares) [134].

extraordinary broad, reflecting the enhanced probability of long dwell times and the increased variance of dwell times at larger values of the intensity for localized waves. It can be well approximated by a double-sided stretched exponential to the power of $1 / 3$ (Fig. 11). The affect of absorption on the form of the measured distributions was not studied.

Notice that, though less probable, negative "delay" times for the multimode case are observed and are also allowed by scattering theory [143, 144]. Negative delay times arise as a result of pulse reshaping [124]. This is in contrast to the "proper" delay times defined as the eigenvalues of the Wigner-Smith matrix $Q=$ $-i S^{*} \cdot \partial S / \partial \omega$ and its trace $\operatorname{Tr} Q=\sum_{a b} W_{a b}=\pi N(\omega)=$ $\tau_{H}$, called the Heisenberg time $\tau_{H}$; the channel-average $\tau_{H} / 2 N=\tau_{W}$ is associated with the Wigner-Smith de-

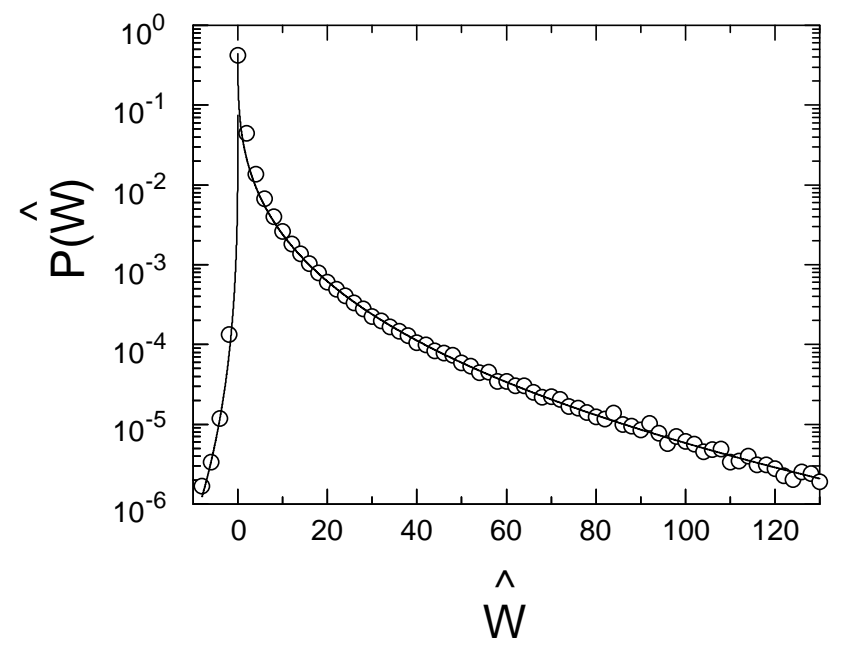

FIG. 11: Probability distribution of the normalized weighted delay time, $P(\hat{W})$, for localized waves (circles) in alumina sample with $L=90 \mathrm{~cm}$. The solid line is the model distribution, $P(\hat{W})=a \exp \left(-b|\hat{W}|^{1 / 3}\right)$, with $a=0.44$ and $b=2.42$ for $\hat{W}>0$, and $a=0.07$ and $b=5.50$ for $\hat{W}<0$. From Ref. 134.

lay time $\tau_{W}$. Substantial theoretical effort in mesoscopic physics has focused on the probability distributions of the "proper" delay times and the Wigner-Smith delay time [145, 146, 147, 148], whose measurements require knowledge of the entire Wigner-Smith matrix. The joint distribution of the inverse "proper" delay times, $\gamma_{n}=1 / \tau_{n}$, was found to be the Laguerre ensemble of random-matrix theory [146], in which the correlation functions of $\tau_{n}$ 's are given in terms of Laguerre polynomials, whereas $P\left(\tau_{W}\right)$ was found to follow universal distributions with algebraic decays in the localized [135, 136, 137] and critical [149, 150] regimes, respectively. It has also been shown that the statistics of delay times relates to the statistics of the eigenfunction intensities and may be used to probe eigenfunction fluctuations in closed mesoscopic samples of any spatial dimension [151, 152.

\section{STATISTICS OF PULSED TRANSMISSION}

In addition to probing the time evolution of average transmission and its increasing suppression, and the statistics of delay time for incident pulses, it is possible to measure the spatial and polarization correlation functions and the full distribution of the field and intensity at any value of $t$ 153]. The cumulant correlation function with displacement and polarization rotation of intensity of the transmitted wave normalized to its ensemble average value for an incident pulse of linewidth $\sigma$ at time $t$ has the form,

$$
C=F+\kappa_{\sigma}(t)(1+F),
$$


where $F$ is the complex square of the field correlation function. The parameter $\kappa_{\sigma}(t)$ establishes the degree of correlation at a delay time $t$ from the center of an exciting pulse of bandwidth $\sigma$, and is evidently the fractional intensity correlation at a point at which $F$ vanishes. This is the same form as the corresponding steady-state correlation functions with the degree of steady state correlation $\kappa_{0}$ instead of $\kappa_{\sigma}(t)$ [154, 155]. The field correlation function is found not to be a function of time, while the degree of intensity correlation $\kappa_{\sigma}(t)$ varies with time and depends upon the pulse bandwidth [153]. This is seen in the polarization measurements shown in Fig. 12 carried out in an ensemble of 12,000 configurations of low density alumina spheres at $L=90 \mathrm{~cm}$ over the frequency range 16.95-17.05 GHz near the peak of the fourth Mie resonance with spectral width $\sigma=5 \mathrm{MHz} 153$. For the conditions given above, $\kappa_{0}=0.29$. The dynamic probability distribution of normalized intensity is found to depend only upon $\kappa_{\sigma}(t)$, which is equal to the variance of the normalized total transmission at a fixed delay time, $\operatorname{var}\left(s_{a}(t)\right)$. The intensity distribution at a fixed delay time is given by Eq. (1) with $g=2 / 3 \kappa$ and closely corresponds to the measured intensity distribution, even for $\kappa \gg 1$. Values of $\kappa_{\sigma}(t)$ much greater than $\kappa_{0}$ are found at long delay times. For an incident pulse width $\sigma=1$ $\mathrm{MHz}$ in a sample with $L=61 \mathrm{~cm}$, in the frequency range 9.95-10.05 GHz, just above the first Mie resonance, at $t=740 \mathrm{~ns}$, we find $\kappa=9.55$.

\section{FIELD STATISTICS}

The isotropic approximation of random matrix theory is equivalent to the assumption that the field distribution in a given configuration is a circular Gaussian [156]. This is demonstrated by measurements of the microwave field in random media, which show that the probability distribution, $P(E)$, of the field normalized to the ensemble average of the field amplitude, $E=t_{a b} / \sqrt{\left\langle\left|t_{a b}\right|^{2}\right\rangle}=$ $r+\sqrt{-1} i$, is given by substituting $r^{2}+i^{2}$ for $s_{a b}$ in Eq. (1) [157]. The probability distribution for the transmitted field for a sub-ensemble with a fixed value of $s_{a}$ is thus Gaussian. Because the field in samples with a fixed value of $s_{a}$ is a Gaussian random process, the field normalized to its average value in a given configuration and the total transmission are statistically independent. The field correlation function may therefore be written as a product of correlators of the normalized Gaussian field and the square root of the total transmission. Since the field correlation function with frequency shift is the Fourier transform of the time-of-flight distribution [29, 30], pulse dynamics, which shows progressive suppression of transport, may be expressed in terms of the spectral correlation function of the normalized Gaussian field and the square root of total transmission. We expect that the correlation function of the Gaussian field will not be af-
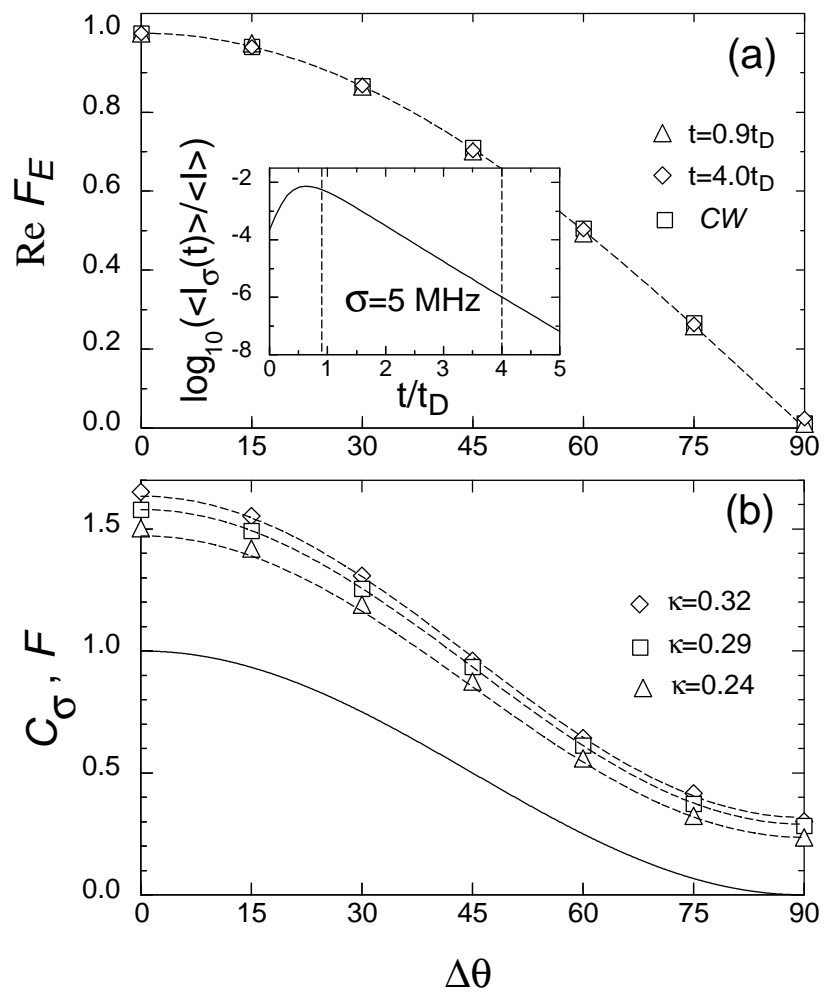

FIG. 12: (a) Real part of the field correlation function, $F_{E}(\Delta \theta)$, and (b) intensity correlation function, $C_{\sigma}(\Delta \theta)$, with polarization rotation of the transmitted wave at the two delay times following pulsed excitation by a Gaussian pulse with spectral width $\sigma=5 \mathrm{MHz}$ and for monochromatic excitation (CW). The dashed curves are (a) $F_{E}(\Delta \theta)=\cos (\Delta \theta)$ and (b) $C_{\sigma}=F(\Delta \theta)+\kappa_{\sigma}(t)[F(\Delta \theta)+1]$, where $F(\Delta \theta)=\left|F_{E}(\Delta \theta)\right|^{2}$ with the values of $\kappa_{\sigma}(t)$ indicated. The solid curve is $F(\Delta \theta)$. The logarithm of the average pulsed transmission through the alumina sample for $\sigma=5 \mathrm{MHz}$, normalized by the average steady-state transmitted intensity, is shown in the inset. The delay times at which correlation is presented in the figure are indicated by vertical dashed lines. From Ref. [153].

fected by mesoscopic fluctuations. On the other hand, the correlation function of the square root of the total transmission reflects mesoscopic fluctuations. In this way the dynamics of the field with the observed suppression of transmission in the time domain due to the growing impact of localization is directly tied to mesoscopic fluctuations.

\section{CONCLUSION}

Because the character of wave transport depends upon the proximity to the localization threshold, signatures of localization, and of the approach to localization, can be found whenever statistical measurements can be made in ensembles of random sample realizations. A large number of statistically different sample configurations may 
arise as a result of constant motion of colloidal systems, or in powdered samples, or in collections of scattering particles that can be tumbled. They may also be created by illuminating different parts of a large slab or by tuning the frequency of a monochromatic source through a spectrum much wider than the correlation frequency. The probability distributions of intensity and total transmission in steady-state and pulsed measurements can be described in terms of the variance of normalized total transmission, which is equal to the degree of intensity correlation, $\operatorname{var}\left(s_{a}\right)=\kappa$. The variation of $\kappa$ with delay time directly charts the increasing impact of localization and is independent of absorption. The decrease of fluctuations and correlation with absorption in steady-state measurements reflects the reduced weight with increasing absorption of long trajectories associated with strong correlation.

Absorption reduces both steady-state and pulsed transmission. The influences of absorption and weak localization can be isolated in the time domain. Absorption reduces the intensity of pulsed transmission exponentially. This reduction can be cancelled in the analysis of data by multiplying by the corresponding growing exponential, to yield the transmitted pulse that would be observed in the absence of absorption. The role of localization in averaged transmission at a given time delay is thus displayed directly. In contrast, absorption and localization affects are mixed in steady-state transmission, and either can lead to an exponential decrease of transmission with sample thickness.

The suppression of the spread of a beam within a sample also gives an indication of localization. This can be observed in the broadening of the peak and in the reduction of the coherent backscattering enhancement factor below 2. Other signatures of localization are observed in measurements of the statistics of the single channel delay time and its correlation with the intensity. Given the many signatures of localization, the state of propagation can be unambiguously determined even in the presence of absorption.

\section{ACKNOWLEDGEMENTS}

We gratefully acknowledge stimulating discussion with Z.Q. Zhang, P. Sebbah, A.A. Lisyansky, B. Hu, B.A. van Tiggelen, and J.H. Li. This work was supported by the National Science Foundation under grants DMR0205186 and DMR0538350.

[1] P.W. Anderson, Phys. Rev. 109, 1492 (1958).

[2] D.J. Thouless, Phys. Rev. Lett. 39, 1167 (1977).

[3] E. Abrahams, P.W. Anderson, D.C. Licciardello, and T.V. Ramakrishnan, Phys. Rev. Lett. 42, 673 (1979).
[4] P.W. Anderson, D.J. Thouless, E. Abrahams, and D.S. Fisher, Phys. Rev. B 22, 3519 (1980).

[5] Mesoscopic Phenomena in Solids, eds. B.L. Altshuler, P.A. Lee, and R.A. Webb, (Elsevier, Amsterdam, 1991).

[6] A. Ioffe and A.R. Regel, Prog. Semicond. 4, 237 (1960).

[7] M.E. Gertsenshtein and V.B. Vasil'ev, Theor. Probab. Appl. 4, 391 (1959).

[8] G. Papanicolaou, J. Appl. Math. 21, 13 (1971).

[9] P.L. Sulem and U. Frisch, J. Plasma Phys. 8, 217 (1972).

[10] S. John, Phys. Rev. Lett. 53, 2169 (1984).

[11] P.W. Anderson, Philos. Mag. 52, 505 (1985).

[12] Scattering and Localization of Classical Waves in Random Media, ed. P. Sheng, (World Scientific Press, Singapore, 1990).

[13] S. John, Physics Today 44, (May, 1991).

[14] A. Lagendijk, in Current Trends in Optics, ed. J.C. Dainty (Academic, London, 1994).

[15] R.A. Webb, S. Washburn, C.P. Umbach, and R.B. Laibowitz, Phys. Rev. Lett. 54, 2696 (1985).

[16] B.L. Altshuler and D.E. Khmelnitskii, JETP Lett. 42, 359 (1985).

[17] P.A. Lee and A.D. Stone, Phys. Rev. Lett. 55, 1622 (1985).

[18] M.P. Van Albada and A. Lagendijk, Phys. Rev. Lett. 55, 2692 (1985).

[19] P.E. Wolf and G. Maret, Phys. Rev. Lett. 55, 2696 (1985).

[20] E. Akkermans, P.E. Wolf, and R. Maynard, Phys. Rev. Lett. 56, 1471 (1986).

[21] D.S. Wiersma, M.P. van Albada, B.A. van Tiggelen, and A. Lagendijk, Phys. Rev. Lett. 74, 4193 (1995).

[22] J.H. Li and A.Z. Genack, Phys. Rev. E 49, 4530 (1994).

[23] A.Z. Genack, Phys. Rev. Lett. 58, 2043 (1987).

[24] M.P. van Albada, B.A. van Tiggelen, A. Lagendijk, and A. Tip, Phys. Rev. Lett. 66, 3132 (1991).

[25] A. Lagendijk and B.A. van Tiggelen, Phys. Rep. 270, 143 (1996).

[26] D. Livdan and A.A. Lisyansky, Phys. Rev. B 53, 14843 (1996).

[27] A.Z. Genack, J.H. Li, N. Garcia, and A.A. Lisyansky, in Photonic Band Gaps and Localization, ed. C.M. Soukoulis, (Plenum, New York, 1993).

[28] G.H. Watson, Jr., P.A. Fleury, and S.L. McCall, Phys. Rev. Lett. 58, 945 (1987).

[29] J.M. Drake and A.Z. Genack, Phys. Rev. Lett. 63, 259 (1989).

[30] A.Z. Genack and J.M. Drake, Europhys. Lett. 11, 331 (1990).

[31] K.M. Yoo, F. Liu, and R.R. Alfano, Phys. Rev. Lett. 64, 2647 (1990); ibid 65, 2210 (1990).

[32] R.H.J. Kop, P. deVries, R. Sprik, and A. Lagendijk, Phys. Rev. Lett. 79, 4369 (1997).

[33] A.A. Chabanov, Z.Q. Zhang, and A.Z. Genack, Phys. Rev. Lett. 90, 203903 (2003).

[34] B. Shapiro, Phys. Rev. Lett. 57, 2168 (1986).

[35] M.P. Van Albada, J.F. de Boer, and A. Lagendijk, Phys. Rev. Lett. 64, 2787 (1990).

[36] P. Sebbah, R. Pnini, and A.Z. Genack, Phys. Rev. E 62, 7348 (2000).

[37] G. Maret and P.E. Wolf, Zeitschrift fur Physik B 65, 409 (1987).

[38] D.J. Pine, D.A. Weitz, P.M. Chaikin, and E. Herbolzheimer, Phys. Rev. Lett. 60, 1134 (1988).

[39] N. Garcia and A.Z. Genack, Phys. Rev. Lett. 63, 1678 
(1989).

[40] J.B. Pendry, Nature 342, 223 (1989).

[41] A.A. Chabanov, M. Stoytchev, and A.Z. Genack, Nature 404, 850 (2000).

[42] M.Ya. Azbel, Solid State Commun. 45527 (1983).

[43] M.Ya. Azbel and P. Soven, Phys. Rev. B 27, 831 (1983).

[44] V. D. Freilikher and S. A. Gredeskul, in Progress in Optics,Volume 30, ed. by E. Wolf, (Elsevier, Amsterdam, 1992), p. 137.

[45] I.M. Lifshits and V.Ya. Kirpichenkov, Sov. Phys. JETP 50, 499 (1979).

[46] J.B. Pendry, J. Phys. C 20, 733 (1987).

[47] J.B. Pendry, Adv. Phys. 43, 461 (1994).

[48] A. MacKinnon and B. Kramer, Phys. Rev. Lett. 47, 1546 (1981)

[49] J.B. Pendry, Nature 351, 438 (1991).

[50] M. Stoytchev and A.Z. Genack, Phys. Rev. B 55, R8617 (1997).

[51] U. Kuhl and H.-J. Stockmann, Physica E 9, 384 (2001).

[52] P. Sebbah, B. Hu, J. Klosner, and A.Z. Genack, submitted for publication.

[53] R. Dalichaouch, J.P. Armstrong, S. Schultz, P.M. Platzman, and S.L. McCall, Nature 354, 53 (1991).

[54] C. Vanneste and P. Sebbah, Phys. Rev. Lett. 87, 183903 (2001).

[55] A.A. Chabanov and A.Z. Genack, Phys. Rev. Lett. 87, 153901 (2001).

[56] M.V. Berry and S. Klein, Eur. J. Phys. 18, 222 (1997).

[57] V. Milner and A.Z. Genack, Phys. Rev. Lett. 94, 073901 (2005).

[58] J. Bertolotti, S. Gottardo, D.S. Wiersma, M. Ghulinyan, and L. Pavesi, Phys. Rev. Lett. 94, 113903 (2005).

[59] S. John, Phys. Rev. Lett. 58, 2486 (1987).

[60] V.P. Bykov, Sov. Phys. JETP 35, 269 (1972).

[61] E. Yablonovitch, Phys. Rev. Lett. 58, 2059 (1987).

[62] A. Cohen, Y. Roth, and B. Shapiro, Phys. Rev. B 38, 12125 (1988).

[63] L.I. Deych, D. Zaslavsky, and A.A. Lisyansky, Phys. Rev. Lett. 81, 5390 (1998).

[64] L.I. Deych, A.A. Lisyansky, and B.L. Altshuler, Phys. Rev. Lett. 84, 2678 (2000).

[65] L.I. Deych, M.V. Erementchouk, and A.A. Lisyansky, Phys. Rev. Lett. 90, 126601 (2003).

[66] L.I. Deych, M.V. Erementchouk, A.A. Lisyansky, and B.L. Altshuler, Phys. Rev. Lett. 91, 096601 (2003).

[67] L.I. Deych, A.A. Lisyansky, and B.L. Altshuler, Phys. Rev. B 64, 224202 (2001).

[68] Y. Asada, K. Slevin, T. Ohtsuki, L.I. Deych, A.A. Lisyansky, and B.L. Altshuler, J. Phys. Soc. Jap. 72A, 173 (2003).

[69] K. Slevin, Y. Asada, and L.I. Deych, Phys. Rev. B 70, 054201 (2004)

[70] J. Prior, A. M. Somoza, and M. Ortuno, Phys. Rev. B 72, 024206 (2005)

[71] A.D. Stone, P.A. Mello, K.A. Muttalib, and J.L. Pichard, in Mesoscopic Phenomena in Solids, eds. B.L. Altshuler, P.A. Lee, and R.A. Webb, (Elsevier, Amsterdam, 1991).

[72] C.W.J. Beenakker, Rev. Mod. Phys. 69, 731 (1997).

[73] M.C.W. van Rossum and Th.M. Nieuwenhuizen, Rev. Mod. Phys. 71, 313 (1999).

[74] A.Z. Genack and A.A. Chabanov, in Wave and Imaging through Complex Media, ed. by P. Sebbah, (Kluwer, Dordrecht, 2001).
[75] R. Landauer, Philos. Mag. 21, 863 (1970).

[76] E.N. Economou and C.M. Soukoulis, Phys. Rev. Lett. 46, 618 (1981).

[77] D.S. Fisher and P.A. Lee, Phys. Rev. B 23, R6851 (1981).

[78] S.K. Cheung, X. Zhang, Z.Q. Zhang, A.A. Chabanov, and A.Z. Genack, Phys. Rev. Lett. 92, 173902 (2004).

[79] A.Z. Genack, Europhys. Lett. 11, 733 (1990).

[80] M.J. Stephen and G. Cwilich, Phys. Rev. Lett. 59, 285 (1987).

[81] P.A. Mello, Phys. Rev. Lett. 60, 1089 (1988).

[82] P.A. Mello, E. Akkermans, and B. Shapiro, Phys. Rev. Lett. 61, 459-462 (1988).

[83] S. Feng, C. Kane, P.A. Lee, and A.D. Stone, Phys. Rev. Lett. 61, 834 (1988).

[84] A.Z. Genack, N. Garcia, and W. Polkosnik, Phys. Rev. Lett. 65, 2129 (1990).

[85] D. Vollhardt and P. Wolfle, Phys. Rev. B 22, 4666 (1980).

[86] N. Garcia and A.Z. Genack, Phys. Rev. Lett. 66, 1850 (1991).

[87] A.Z. Genack and N. Garcia, Phys. Rev. Lett. 66, 2064 (1991).

[88] D.S. Wiersma, P. Bartolini, A. Lagendijk, and R. Righini, Nature 390, 671 (1997).

[89] F. Scheffold, R. Lenke, R. Tweer, and G. Maret, Nature 389, 206 (1999).

[90] R.L. Weaver, Phys. Rev. B 47, 1077 (1993).

[91] E. Kogan and M. Kaveh, Phys. Rev. B 52, R3813 (1995).

[92] Th.M. Nieuwenhuizen and M.C.W. van Rossum, Phys. Rev. Lett. 74, 2674 (1995).

[93] M. Stoytchev and A.Z. Genack, Phys. Rev. Lett. 79, 309 (1997).

[94] P.M. Morse and H. Feshbach, Methods of Theoretical Physics, (McGraw-Hill, New York, 1953).

[95] A. Lagendijk, R. Vreeker, and P. DeVries, Phys. Lett. A 136, 81 (1989).

[96] J. X. Zhu, D. J. Pine, and D. A. Weitz, Phys. Rev. A 44, 3948 (1991).

[97] K. Bodland, J. Sound Vib. 73, 19 (1980).

[98] F. Kawakami and K. Yamiguchi, J. Acoust. Soc. Am. 80, 543 (1986).

[99] J. Burkhardt and R.L. Weaver, J. Acoust. Soc. Am. 100, 320 (1996).

[100] O.L. Lobkis, R.L. Weaver, and I. Rozhkov, J. Sound Vib. 237, 281 (2000).

[101] E. Doron, U. Smilansky, and A. Frenkel, Phys. Rev. Lett. 65, 3072 (1990).

[102] H. Alt, H.-D. Graf, H.L. Harney, R. Hofferbert, H. Lengeler, A. Richter, P. Schardt, and H.A.Weidenmuller, Phys. Rev. Lett. 74, 62 (1995).

[103] B.L. Altshuler, V.E. Kravtsov, and I.L. Lerner, in Mesoscopic Phenomena in Solids, eds. B.L. Altshuler, P.A. Lee, and R.A. Webb, (Elsevier, Amsterdam, 1991).

[104] B.A. Muzykantskii and D.E. Khmelnitskii, Phys. Rev. B 51, 5480 (1995).

[105] A.D. Mirlin, Phys. Rep. 326, 259 (2000).

[106] K.B. Efetov, Adv. Phys. 32, 53 (1983).

[107] S.E. Skipetrov and B.A. van Tiggelen, Phys. Rev. Lett. 92, 113901 (2004).

[108] S.K. Cheung and Z.Q. Zhang, cond-mat/0509381 (2005)

[109] S.E. Skipetrov and B.A. van Tiggelen, 
cond-mat/0508726) (2005).

[110] N. Lawandy, R. Balachandran, A. Gomes, and E. Sauvain, Nature 368, 436 (1994).

[111] A.Z. Genack and J.M. Drake, Nature 368, 400 (1994).

[112] H. Cao, Y.G. Zhao, S.T. Ho, E.W. Seelig, Q.H. Wang, and R.P.H. Chang, Phys. Rev. Lett. 82, 2278 (1999).

[113] S.V. Frolov, Z.V. Vardeny, and K. Yoshino, Phys. Rev. B 57, 9141 (1999).

[114] X.Jiang and C.M. Soukoulis, Phys. Rev. Lett. 85, 70 (2000).

[115] V.M. Apalkov, M.E. Raikh, and B. Shapiro, Phys. Rev. Lett. 89, 016802 (2002).

[116] A. Yamilov and H. Cao, Phys. Rev. A 69, 031803(R) (2004).

[117] V.I. Kopp, B. Fan, H.K.M. Vithana, and A.Z. Genack, Opt. Lett. 23, 1707 (1998).

[118] P.V. Shibaev, V.I. Kopp, and A.Z. Genack, Jour. Phys. Chem. B 107, 6961 (2003).

[119] J. Schmidtke, W. Stille, and H. Finkelmann, Phys. Rev. Lett. 90, 083902 (2003).

[120] V.I. Kopp and A.Z. Genack, Phys. Rev. Lett. 89, 033901 (2002).

[121] M. Stoytchev and A.Z. Genack, Opt. Lett. 24, 262 (1999).

[122] A.A. Chabanov and A.Z. Genack, in Wave Scattering in Complex Media: from Theory to Applications, eds. B.A. van Tiggelen and S. Skipetrov, (Kluwer Academic, Dordrecht, 2003).

[123] P. Sebbah, O.Legrand, B.A. van Tiggelen, and A.Z. Genack, Phys. Rev. E 56, 3619 (1997).

[124] P. Sebbah, O. Legrand, and A.Z. Genack, Phys. Rev. E 59, 2406 (1999).

[125] J.H. Li, Interfacial scattering and microparticle resonances in random media, Ph.D. thesis, City University of New York, (1993).

[126] K. Busch and C. M. Soukoulis, Phys. Rev. B 54, 893 (1996).

[127] A.Z. Genack, A.A. Chabanov, P. Sebbah, and B.A. van Tiggelen, in Wave Scattering in Complex Media: from Theory to Applications, eds. B.A. van Tiggelen and S. Skipetrov, (Kluwer Academic, Dordrecht, 2003).

[128] C.W.J. Beenakker, in Photonic Crystals and Light Localization in the 21st Century, ed. C.M. Soukoulis, (Kluwer Acadenic, Dordrecht, 2001).

[129] I. Freund, Waves in Random Media 8, 119 (1998).

[130] A.Z. Genack, P. Sebbah, M. Stoytchev, and B.A. van Tiggelen, Phys. Rev. Lett. 82, 715 (1999).

[131] B.A. van Tiggelen, P. Sebbah, M. Stoytchev, and A.Z. Genack, Phys. Rev. E 59, 7166 (1999).

[132] H. Schomerus, K.J.H. van Bemmel, and C.W.J. Beenakker, Phys. Rev. E 63, 026605 (2001).
[133] A. Lagendijk, J. Gomez-Rivas, A. Imhof, F.J.P. Schuurmans, and R. Sprik, in Photonic Crystals and Light Localization in the 21st Century, ed. C.M. Soukoulis, (Kluwer Academic, Dordrecht, 2001).

[134] A.A. Chabanov and A.Z. Genack, Phys. Rev. Lett. 87, 233903 (2001).

[135] C. Texier and A. Comtet, Phys. Rev. Lett. 82, 4220 (1999); A. Comtet and C. Texier, J. Phys. A 30, 8017 (1997).

[136] C.J. Bolton-Heaton, C.J. Lambert, V.I. Falko, V. Prigodin, and A.J. Epstein, Phys. Rev. B 60, 10569 (1999).

[137] A.Ossipov, T. Kottos, and T. Geisel, Phys. Rev. B 61, 11411 (2000).

[138] B.L. Altshuler and V.N. Prigodin, JETP Lett. 47, 43 (1988).

[139] Y.V. Fyodorov, JETP Lett. 78, 250 (2003).

[140] H. Schomerus, Phys. Rev. E 64, 026606 (2001).

[141] R.G. Newton, Scattering Theory of Waves and Particles, (Springer-Verlag, New York, 1982), Sec. 11.13.

[142] B.A. van Tiggelen and E. Kogan, Phys. Rev. A 49, 708 (1994).

[143] E.P. Wigner, Phys. Rev. 98, 145 (1955).

[144] F.T. Smith, Phys. Rev. 118, 349 (1960).

[145] Y.V. Fyodorov and H.J. Sommers, J. Math. Phys. 38, 1918 (1997).

[146] P.W. Brouwer, K.M. Fram, and C.W.J. Beenakker, Phys. Rev. Lett. 78, 4737 (1997).

[147] C.W.J. Beenakker and P.W. Brouwer, Physica E 9, 463 (2001).

[148] H.J. Sommers, D.V. Savin, and V.V. Sokolov, Phys. Rev. Lett. 87, 094101 (2001).

[149] F. Steinbach, A. Ossipov, T. Kottos, and T. Geisel, Phys. Rev. Lett. 85, 4426 (2000).

[150] T. Kottos and M. Weiss, Phys. Rev. Lett. 89, 056401 (2002).

[151] A. Ossipov and Y.V. Fyodorov, Phys. Rev. B 71, 125133 (2005).

[152] J.A. Mendez-Bermudez and T. Kottos, Phys. Rev. B 72, 064108 (2005).

[153] A.A. Chabanov, B. Hu, and A.Z. Genack, Phys. Rev. Lett. 93, 123901 (2004).

[154] P. Sebbah, B. Hu, A.Z. Genack, R. Pnini and B. Shapiro, Phys. Rev. Lett. 88, 123901 (2002).

[155] A.A. Chabanov, N.P. Tregoures, B.A. van Tiggelen, and A.Z. Genack, Phys. Rev. Lett. 92, 173901 (2004).

[156] J.W. Goodman, Statistical Optics, (John Wiley, New York, 2000).

[157] A.A. Chabanov and A.Z. Genack, cond-mat/0502334 (2005). 